\title{
Recursos humanos e eficiência: um estudo em hospitais brasileiros de pequeno porte
}

Human resources and efficiency: a study in Brazilian small hospitals

\author{
Júnia Marçal Rodrigues (1) \\ Allan Claudius Queiroz Barbosa (2) \\ (1) Unimed-BH \\ (2) Universidade Federal de Minas Gerais
}

\begin{abstract}
The paper examined the extent to which Human Resource Management (HRM) contributes to the performance of small Brazilian hospitals from an efficiency perspective. The literature review addressed efficiency to assess hospital performance by measuring the contribution of Human Resources (HR) in health and industrial organizations. The methodological pathway used Data Envelopment Analysis (DEA) on a sample of 702 hospitals from secondary data from census surveys with 2777 small hospitals. The central hypothesis of the study, that GRH contributes to the efficiency of hospitals, was confirmed by the results considering that this was the variable with the greatest need for improvement in the efficiency of small hospitals. Other variables were also relevant in the context of hospital efficiency, such as the influence of peculiarities regarding size (number of beds), legal nature of institutions and regional distribution.
\end{abstract}

\section{Keywords}

hospital performance, human resource management, efficiency and health.

JEL Codes I1, I10.

\section{Resumo}

Este artigo verificou em que medida a Gestão de Recursos Humanos (GRH) contribui para o desempenho de hospitais de pequeno porte brasileiros na perspectiva da eficiência. A revisão de literatura tratou da eficiência para avaliar o desempenho hospitalar mensurando a contribuição de Recursos Humanos (RH) em organizações industriais e de saúde. O percurso metodológico utilizou Análise Envoltória de Dados (DEA) numa amostra de 702 hospitais a partir de dados secundários oriundos de levantamento censitário com 2.777 hospitais de pequeno porte. A hipótese central do estudo, de que a GRH contribui para a eficiência de hospitais, foi confirmada pelos resultados, considerando que essa foi a variável com maior necessidade de incremento para melhoria da eficiencia dos hospitais de pequeno porte. Outras variáveis também mostraram-se relevantes no contexto da eficiência hospitalar, tais como a influência das particularidades quanto ao porte (número de leitos), a natureza jurídica das instituições e a distribuição regional.

\section{Palavras-chave}

desempenho hospitalar, gestão de recursos humanos, eficiência, saúde.

Códigos JEL I1, I10. 


\title{
1 Introdução
}

A gestão de recursos humanos (GRH) possui o desafio de avaliar sua vinculação aos resultados organizacionais. Para as instituições de saúde, em especial os hospitais, as múltiplas variáveis que estão associadas ao seu resultado evidenciam a complexidade na busca de caracterizar a contribuição de qualquer uma em específico. Rodrigues et al. (2015) afirmam que

\begin{abstract}
esta questão suscita a ideia da busca pela mensuração da gestão de recursos humanos como tema recorrente na literatura especializada, frequentemente associada à perspectiva estratégica num contexto de resultados multideterminados por fatores diversos com intensidade diferenciada, conforme a realidade de cada organização. De outro, a relevância do equipamento hospitalar, entre os serviços de saúde, pode ser caracterizada pelo uso intensivo da força de trabalho, tipo de assistência prestada, nivel de complexidade dos procedimentos realizados, localização, porte (capacidade instalada), integração com a rede de atenção à saúde, além do considerável volume de recursos financeiros (p. 1.129).
\end{abstract}

Nesse contexto, os hospitais são caracterizados como organizações sociais complexas (Glouberman, 2001), em função da necessária articulação entre a dimensão assistencial e a dimensão da gestão, cada uma com demandas bem definidas e convergentes quanto aos fins, embora nem sempre alinhadas quanto à aplicação dos meios para cumprir os objetivos, com lacunas que acabam por fragilizar ambas. Somem-se a isso a tecnologia e todos os insumos presentes na rotina de um hospital, o extenso aparato jurídico e normativo que delimita tanto a atuação dos profissionais da saúde (através, por exemplo, dos conselhos de classe e outras instâncias de representação) e a natureza dos procedimentos realizados, bem como a forma de organização e distribuição dos diferentes serviços de saúde que influenciam diretamente na performance das instituições hospitalares.

A relação entre performance organizacional e GRH é vista quando os resultados assistenciais são atingidos pela competência dos profissionais aplicando conhecimento e pela existência de tecnologia e recursos, devendo ser usados de forma eficiente para minimizar riscos aos pacientes e sempre que possível aumentar sua satisfação (Zigan et al., 2008).

Torna-se importante delimitar quais ou como os fatores da GRH estão associados ao desempenho hospitalar. Essa perspectiva de análise constitui o eixo central para o desenvolvimento deste artigo e remete a um campo multidisciplinar que demanda a interface entre diversas áreas do conhecimento, como relações industriais, psicologia industrial e organizacional, economia, sociologia e outras (Huselid, 1995; Paauwe, 2009). 
O desempenho é equivalente à ideia de eficiência para a WHO (World Health Organization). Isto é, segundo Rodrigues et al. (2015) e Purbey, Mukherjee e Bhar, (2007), "uma função da contribuição do sistema para os objetivos intrínsecos e os inputs utilizados para atingi-los, o que significa dimensões que conduzem ao desempenho e aquelas que demonstram parâmetros de efetividade" (p. 1.130).

A eficiência está pautada na oferta dita "ótima" de bens e serviços de saúde para o consumidor ou usuário dos serviços ao menor custo possível (eficiência técnica e alocativa ${ }^{1}$ ) em um mercado (saúde) caracterizado por economias de escala e escopo) com barreiras de entrada. Ao analisar a eficiência no âmbito hospitalar, Andrade e Maia (2007) ressaltam que a principal dificuldade decorre da diversidade do produto ofertado entre hospitais. Em qualquer contexto, é indiscutível ser a eficiência uma dimensão básica a ser avaliada quando se trata de estudar desempenho hospitalar (Lins et al., 2007).

De forma geral os estudos abordam de um lado pela perspectiva econômica, através do uso de funções paramétricas que determinam eficiência econômica absoluta em contraposição ao benchmarking; e de outro, por programas matemáticos não paramétricos através da avaliação de eficiência de forma comparativa entre organizações. Considerando que a primeira perspectiva pode ser de difícil exequibilidade por requerer uma amostra ampla e a especificação das funções eficiência/ineficiência. Por isso, a maioria dos estudos que visam mensurar a eficiência utilizam modelos matemáticos, notadamente o Data Envelopment Analysis (DEA) (Weng et al., 2009).

Existe relativo consenso de que essas ferramentas são efetivas na mensuração da eficiência em organizações de saúde, em geral, com sua aplicação, principalmente nos Estados Unidos desde a década de 1980 (Grosskopf; Valdmanis; 1987). A eficiência, através do DEA, está relacionada ao fato de que o aumento de um output requer a redução, no mínimo, em outro output ou aumento em pelo menos um input. Como alternativa, a redução de um input requer necessariamente o aumento de pelo menos outro input ou a redução de, no mínimo, um output. A relação dos insumos com o custo é a dimensão econômica que busca responder à pergunta: "o

1 Eficiência técnica: foco em minimizar insumos para produção. Eficiência alocativa: recursos alocados de modo a produzir em nível ótimo. Outra medida de eficiência é a de custo-efetividade, que é alcançada quando a produção está organizada de forma a minimizar o custo de produzir determinado nível de produto. 
quanto economizamos na aquisição de insumos?" A relação dos produtos com os insumos é definida como a eficiência e faz referência a "quão produtivamente utilizamos nossos recursos" (Magnussen, 1996).

$\mathrm{Na}$ utilização do método DEA se a mensuração do erro acontece em uma organização eficiente, isso vai afetar a construção da fronteira de eficiência. Ao contrário, se o erro ocorre em uma organização ineficiente, a sua mensuração pode ser isolada para essa organização. Outra questão diz respeito à especificação das variáveis, uma vez que qualquer organização da amostra pode parecer eficiente de acordo com os inputs e output que foram considerados. E, ainda, a distribuição da eficiência dependerá não somente da definição dos outputs, mas também da quantidade de inputs e outputs considerados (Sexton et al., 1986; Magnussen, 1996).

Ainda sobre a mensuração da eficiência dos cuidados de saúde, uma revisão, feita por Hussey et al. (2009) de artigos publicados entre 1990 e 2008, aponta que a concentração dos indicadores está voltada para os custos não associados à qualidade dos cuidados, com predominância da avaliação da eficiência de hospitais com indicadores como tempo médio de permanência e os custos, ambos ajustados em função do casemix (61\% dos trabalhos). Pouca ênfase na eficiência dos médicos, consultas efetuadas, recursos utilizados na produção realizada, horas médicas, exames, medicamentos e eficiência da equipe de enfermagem. Nenhum artigo versava sobre a eficiência dos cuidados de saúde em âmbito nacional.

Machado e Kuchenbecker (2007) identificam que há uma vasta gama de estudos que sinalizam o processo de transformação dos hospitais com o intuito de adaptá-los às mudanças e desafios vivenciados pelos sistemas de saúde nos últimos anos. O presente estudo, originário de Rodrigues (2016), foi realizado a partir de dados coletados por Facchini, Barbosa et al. (2014) em levantamento nacional censitário por meio de survey in loco de todos os hospitais de pequeno porte (HPP) brasileiros.

\section{Método}

Neste estudo foram utilizados dados de levantamento in loco (através de survey) realizado por Facchini, Barbosa et al. (2014) ${ }^{2}$ junto aos 3.524 hospipossuía em 20133.524 estabelecimentos hospitalares de pequeno porte, públicos e privados, 
tais de pequeno porte ${ }^{3}$ brasileiros, entre os meses de janeiro e novembro de 2014. Através de respostas autodeclaradas pelos membros do corpo diretivo (diretor clínico, diretor-geral ou chefe da enfermagem), teve como base a definição das seguintes dimensões, adaptadas a partir de Veillard et al. (2005): a) Infraestrutura: existência de dados selecionados da base Cadastro Nacional de Estabelecimentos de Saúde (CNES) referentes aos aspectos jurídicos/legais, físicos, equipamentos, administrativos. A partir das fichas de cadastramento do CNES, realizou-se a seleção das variáveis a serem confirmadas; b) Assistencial: presença de ambulatórios, urgência, internação, serviços de apoio diagnósticos, leitos, clínicas, especialidades, regulação assistencial, presença de profissionais, turnos de trabalho e outros que caracterizem a assistência prestada, origem da clientela, e dados de ocupação de leitos; c) Recursos Humanos: categorias profissionais existentes, tipos de contrato informado, carga horária e outros quesitos a partir do perfil profissional do estabelecimento e informado no CNES; d) Financiamento: fontes regulares e extraordinárias de financiamentos de origem municipal, estadual e federal, além de dados referentes às despesas de cada instituição; e) Qualidade e Segurança do Paciente: quesitos de verificação em relação a: qualificação profissional, estratégias e planos de ação voltados para a qualidade da atenção prestada e a segurança do paciente. A partir do perfil assistencial do estabelecimento foram coletadas informações que permitem inferir sobre a qualidade do serviço assistencial; f) Responsabilidades na Rede de Atenção à Saúde Loco-Regional: informação sobre responsabilidades junto aos serviços loco-regionais, inclusive relação com os gestores, instrumento, relação com regulação municipal/ estadual, Conselho de Saúde e outros.

Utilizou-se a Análise Envoltória dos Dados (DEA) com o objetivo de determinar a eficiência dos hospitais através da comparação entre eles a par-

sendo que 2.724 ofereciam leitos ao SUS.

3 López (2004) utiliza como referência que esses estabelecimentos possuem pelo menos cinco leitos disponíveis para internação de pacientes capazes de garantir atendimento básico de diagnóstico e tratamento, com equipe clínica organizada e com prova de admissão e assistência permanente de médicos. Segundo esses mesmos princípios, um hospital de pequeno porte exige a presença de serviço de enfermagem e atendimento terapêutico direto ao paciente durante 24 horas, com serviços de laboratório e radiologia, cirurgia e/ou parto, além de registros médicos organizados para observação e acompanhamento rápido dos casos. A Comissão Intergestora Tripartite (CIT), espaço intergovernamental, político e técnico onde acontece o planejamento, negociação e implementação das políticas de saúde pública, estabeleceu em 2013 que esses hospitais estariam na faixa de 0 a 50 leitos. 
tir de certa homogeneidade quanto às tarefas realizadas, objetivos, condições de mercado e autonomia na tomada de decisões e que se diferenciam nas quantidades dos recursos consumidos (inputs) e das saídas produzidas (outputs), em função de aspectos internos (Cook et al. 2009).

Os principais modelos de análise envoltória de dados são: a) Modelo $C C R,{ }^{4}$ conhecido como CRS (Constant Returns to Scale). É um modelo de retornos constantes de escala, ou seja, qualquer variação nas entradas (inputs) produz variação proporcional nas saídas (outputs) (Cook; Seiford, 2009). Quando orientado a inputs, cada Decision Making Unit (DMU) pode escolher os pesos para as variáveis (entrada ou saída), desde que não gere uma razão superior a 1 para as outras DMUs. A estrutura matemática desses modelos permite que uma DMU seja considerada eficiente com vários conjuntos de pesos. Quando orientado a outputs, maximiza as saídas (outputs) sem nenhuma alteração nas entradas (inputs). Indica fator pelo qual todos os produtos devem ser multiplicados para que a DMU possa atingir a fronteira eficiente (Jacobs, 2001); b) Modelo BCC ${ }^{5}$ ou VRS (Variable Returns to Scale), permite que as DMUs com baixos inputs tenham retornos crescentes de escala e aquelas com altos inputs tenham retornos decrescentes de escala.

No modelo orientado a inputs e outputs, Jacobs (2001) compara classificações de eficiência e aponta duas possibilidades: a) Inputs - quando positivos, representam retornos crescentes de escala; quando negativos, representam retornos decrescentes de escala; e caso sejam nulos, representam retornos constantes de escala; b) Outputs - indicam retornos decrescentes de escala, quando positivos; retornos crescentes de escala, quando negativos, e retornos constantes de escala, quando nulos (Jacobs, 2001).

Para análise da eficiência o conjunto das DMUs, os hospitais aqui estudados devem ter a mesma utilização de entradas e saídas, com variação apenas em intensidade. A eficiência de um hospital é sempre relativa aos demais hospitais que fazem parte da amostra do presente estudo. Pela aplicação da modelagem DEA cada observação individual é otimizada para possibilitar o cálculo de uma fronteira de eficiência, determinada pelas unidades que são Pareto eficientes (quando, e somente se, uma unidade não consegue melhorar alguma de suas características sem piorar as de-

4 Sigla para as iniciais dos seus autores desenvolvedores: Charnes, Cooper e Rhodes.

5 Sigla para as iniciais dos seus autores desenvolvedores: Banker, Charnes e Cooper. 
mais). De forma que o máximo que poderia ter sido produzido é obtido por meio da observação das unidades mais produtivas (Mello et al., 2005). Ressalva a ser feita é a natureza determinística da técnica, que pode ter os resultados da eficiência afetados por ruídos nos dados.

A escolha das variáveis de entradas e saídas (indicadores) expressa a categorização das variáveis a partir do instrumento aplicado no censo de hospitais de pequeno porte. O agrupamento das variáveis do instrumento de pesquisa teve como referência as categorias demonstradas por Donabedian $(1980 ; 1992)$ para avaliação da qualidade dos serviços de saúde: a) Estrutura: recursos humanos, físicos e financeiros necessários ao fornecimento de cuidado em instituições de saúde. A estrutura pode contribuir para maximizar ou minimizar o desempenho, embora poucos estudos colaboram para elucidar essa associação; b) Processo: práticas de cuidado efetivamente realizadas entre os profissionais de saúde e os pacientes; c) Resultado: modificações no estado de saúde dos pacientes atribuíveis às ações de cuidado, podendo ser incluídos aspectos fisiológicos, físicos, psicológicos, sociais e avaliativos.

Os Quadros 1 e 2 apresentam as variáveis do instrumento de coleta de dados, aplicado no levantamento censitário junto aos HPPs, de acordo com as respectivas categorias e dimensões de estrutura (sete categorias e 44 variáveis) e processo (seis categorias e 50 variáveis) utilizadas para aplicação do modelo de eficiência. A escolha das variáveis para o modelo teve como critério a ocorrência de resposta válida que, exceto o número de leitos (quantidade), corresponde à ocorrência de cada variável na realidade do hospital de pequeno porte.

\section{Quadro 1 Categorias e variáveis da dimensão estrutura}

\begin{tabular}{l|l}
\hline Categoria & Variáveis \\
\hline Equipamentos & Equipe móvel \\
\cline { 2 - 2 } & Incubadora \\
\cline { 2 - 2 } & Monitor de ECG \\
\cline { 2 - 2 } & Monitor de pressão invasivo \\
\cline { 2 - 2 } & Monitor de pressão não invasivo \\
\cline { 2 - 2 } & Otoscópio \\
\cline { 2 - 2 } & Oxímetro \\
\cline { 2 - 2 } & Raio Xaté $110 \mathrm{~mA}$ \\
\cline { 2 - 2 }
\end{tabular}

(continua) 
Quadro 1 (continuação)

\begin{tabular}{|c|c|}
\hline Categoria & Variáveis \\
\hline \multirow[t]{3}{*}{ Equipamentos } & Raio $X$ de 100 a $500 \mathrm{~mA}$ \\
\hline & Refrigerador para vacina e para medicamentos \\
\hline & Respirador/ventilador - adulto \\
\hline \multirow[t]{6}{*}{$\begin{array}{l}\text { Força de } \\
\text { trabalho }\end{array}$} & $\begin{array}{l}\text { Compartilhamento de médicos do hospital com a atenção básica ou outros } \\
\text { pontos da rede }\end{array}$ \\
\hline & Disponibilidade de médicos para a realização de interconsultas \\
\hline & Equipe de atenção da urgência é a mesma da atenção nas enfermarias \\
\hline & Número de profissionais/leito \\
\hline & Presença do profissional médico na urgência/emergência em dias úteis \\
\hline & Presença do profissional médico na urgência/emergência nos finais de semana \\
\hline \multirow{5}{*}{$\begin{array}{l}\text { Mecanismos } \\
\text { de gestão }\end{array}$} & Monitora indicador de custo/internação \\
\hline & Sistema de gestão próprio \\
\hline & $\begin{array}{l}\text { Programação física do SAI/SUS é realizada com a participação da gestão } \\
\text { do hospital }\end{array}$ \\
\hline & $\begin{array}{l}\text { Programação física (SIHD)/SUS é realizada com a participação da gestão } \\
\text { do hospital }\end{array}$ \\
\hline & Auditoria hospitalar \\
\hline Número de leitos & Número total de leitos \\
\hline \multirow[t]{7}{*}{ Políticas GRH } & Desenvolve ações de avaliação de desempenho \\
\hline & Projetos de educação permanente \\
\hline & Saúde do trabalhador \\
\hline & Dispositivo de valorização do trabalhador \\
\hline & Registro de indicadores de gestão de recursos humanos \\
\hline & Desenvolve ações de treinamento e desenvolvimento \\
\hline & Sala de cirurgia ambulatorial \\
\hline \multirow{9}{*}{$\begin{array}{l}\text { Salas de } \\
\text { atendimento }\end{array}$} & Sala de curativo \\
\hline & Sala de enfermagem \\
\hline & Sala de gesso \\
\hline & Sala de medicação \\
\hline & Sala de pequenas cirurgias (sutura) \\
\hline & Sala de repouso/observação adulto \\
\hline & Sala de repouso/observação pediátrico \\
\hline & Quarto de isolamento \\
\hline & Recuperação pós-anestésica \\
\hline
\end{tabular}


Quadro 1 (continuação)

\begin{tabular}{l|l}
\hline Categoria & Variáveis \\
\hline \multirow{3}{*}{$\begin{array}{l}\text { Urgência e } \\
\text { emergência }\end{array}$} & Carrinho de emergência \\
\cline { 2 - 2 } & Desfibrilador \\
\cline { 2 - 2 } & Reanimador pulmonar (AMBU) adulto \\
\cline { 2 - 2 } & Reanimador pulmonar (AMBU) pediátrico \\
\cline { 2 - 2 } & Sala de emergência adulto \\
\hline
\end{tabular}

Fonte: Elaboração própria, a partir de Facchini, Barbosa et al. (2014).

Quadro 2 Categorias e variáveis da dimensão processo

\begin{tabular}{|c|c|}
\hline Categoria & Variáveis \\
\hline \multirow[t]{6}{*}{ Dispositivos } & Dispositivo de acessibilidade \\
\hline & Dispositivo de acolhimento \\
\hline & Dispositivo de acompanhamento da gestante \\
\hline & Dispositivo de acompanhamento 24 horas para idosos \\
\hline & Dispositivo de horário de visita ampliado para além de 1 hora \\
\hline & Dispositivo de visita aberta \\
\hline \multirow{7}{*}{$\begin{array}{l}\text { Monitoramento } \\
\text { de indicadores } \\
\text { assistenciais }\end{array}$} & Satisfação do usuário \\
\hline & Taxa de cesárea \\
\hline & Taxa de infecção hospitalar \\
\hline & Taxa de infecção pós-cirúrgica \\
\hline & Taxa de mortalidade institucional \\
\hline & Taxa de ocupação hospitalar \\
\hline & Tempo médio de permanência \\
\hline \multirow{12}{*}{$\begin{array}{l}\text { Processo } \\
\text { de trabalho/ } \\
\text { procedimentos }\end{array}$} & Administração de antibiótico profilático antes das cirurgias \\
\hline & Adoção de cuidados com a posição do paciente e mudanças de decúbito \\
\hline & Avaliação diária da pele em relação ao surgimento de lesões \\
\hline & Checagem da identificação do paciente a ser operado \\
\hline & Comissão de Controle de Infecção Hospitalar \\
\hline & Corrida de leito multiprofissional \\
\hline & Dupla checagem ao preparar, dispensar e administrar medicamentos \\
\hline & Gestão de risco \\
\hline & Identificação do paciente em pulseira \\
\hline & Identificação visual de pacientes com risco de quedas \\
\hline & Normas e rotinas assistenciais \\
\hline & Organização do trabalho da enfermagem \\
\hline
\end{tabular}


Quadro 2 (continuação)

\begin{tabular}{l|l}
\hline Categoria & Variáveis \\
\hline Processo & Padronização da prescrição de drogas \\
\cline { 2 - 2 } procedimentos & Passagem de plantão médico presencial \\
\cline { 2 - 2 } & Pratica das diretrizes de acolhimento \\
\cline { 2 - 2 } & Prontuário único \\
\cline { 2 - 2 } & Segurança do paciente \\
\hline \multirow{2}{*}{ Regulação } & Regulação de atendimento ambulatorial geral \\
\cline { 2 - 2 } & Regulação de exames \\
\cline { 2 - 2 } & Regulação de internação \\
\cline { 2 - 2 } & Regulação de urgências SAMU 192 \\
\cline { 2 - 2 } & Rede Cegonha \\
\cline { 2 - 2 } & Referência para a UPA 24 horas \\
\cline { 2 - 2 } & Atua de forma conjunta com a atenção básica \\
\hline \multirow{5}{*}{ Serviços } & Ambulatório de especialidades \\
\cline { 2 - 2 } & Cirurgia ambulatorial \\
\cline { 2 - 2 } & Cirurgias de maior complexidade \\
\cline { 2 - 2 } & Urgência/Emergência \\
\cline { 2 - 2 } & Realiza internações \\
\cline { 2 - 2 } & Atenção psicossocial \\
\cline { 2 - 2 } & Serviço de fisioterapia \\
\cline { 2 - 2 } & Serviço de fonoaudiologia \\
\cline { 2 - 2 } & Serviço de imagem \\
\cline { 2 - 2 } & Serviço de laboratório \\
\hline \multirow{2}{*}{ Serviço de terapia ocupacional próprio de material esterilizado móvel de urgência SAMU } \\
\hline
\end{tabular}

Fonte: Elaboração própria, a partir de Facchini, Barbosa et al. (2014).

Q Quadro 3 apresenta as categorias utilizadas para o modelo de eficiência com a respectiva classificação (input ou output).

$\bigcirc$ tratamento dos dados para o modelo de eficiência, com base nos pressupostos teóricos e metodológicos foi realizado através de software para a análise de DEA (Banxia Frontier Analyst). Os hospitais que apresentaram quantidade substancial de dados faltantes em relação aos inputs e outputs selecionados foram excluídos, devido às restrições do método DEA quanto a valores faltantes ou iguais a zero. Do universo de 3.524 
hospitais visitados, $2.777(78,80 \%)$ responderam ao estudo. Pelo modelo aditivo de retornos constantes de escala (orientação para inputs e outputs), $702(25,27 \%)$ deste total permaneceram para análise dos resultados.

Quadro 3 Dimensões e categorias para avaliara eficiência dos hospitais de pequeno porte

\begin{tabular}{l|l|c|c}
\hline \multirow{2}{*}{ Dimensão } & Categorias & Input & Output \\
\hline \multirow{4}{*}{ Estrutura } & Equipamentos & $\mathrm{x}$ & \\
\cline { 2 - 5 } & Força de trabalho & $\mathrm{x}$ & \\
\cline { 2 - 5 } & Mecanismo de gestão & $\mathrm{x}$ & \\
\cline { 2 - 5 } & Número de leitos & $\mathrm{x}$ & \\
\cline { 2 - 5 } & Política de recursos humanos & $\mathrm{x}$ & \\
\cline { 2 - 5 } & Salas de atendimento & $\mathrm{x}$ & \\
\cline { 2 - 5 } & Urgência e emergência & $\mathrm{x}$ & \\
\hline \multirow{5}{*}{ Processo } & Dispositivos & $\mathrm{x}$ & \\
\cline { 2 - 5 } & Monitoramento de indicadores assistenciais & $\mathrm{x}$ & \\
\cline { 2 - 5 } & Processo de trabalho & $\mathrm{x}$ & \\
\cline { 2 - 5 } & Regulação & $\mathrm{x}$ & \\
\cline { 2 - 5 } & Serviços especializados & & $\mathrm{x}$ \\
\cline { 2 - 5 } & Serviços ofertados & x & $\mathrm{x}$ \\
\hline \multirow{2}{*}{ Resultados } & Autorização de Internação Hospitalar (AlH) (frequência) & & \\
\cline { 2 - 5 } & Autorização de Internação Hospitalar (AlH) (valor médio) & & \\
\cline { 2 - 5 } & Total de procedimentos & & \\
\hline
\end{tabular}

Fonte: Elaboração própria.

\section{Resultados}

O resultado do modelo de eficiência, conforme Tabela 1, demonstra que $46,87 \%$ dos hospitais (329 unidades) atingiram a fronteira de eficiência, com predomínio das regiões Norte e Sudeste (62,50\% e 56,16\% respectivamente). 53,13\% (373 unidades) dos hospitais, comparativamente pela fronteira do modelo aplicado, foram considerados ineficientes com maior incidência para as regiões Centro-Oeste e Sul (58,46\% e 63,57\%, respectivamente). Somente as regiões Norte e Sudeste possuem mais de $50 \%$ dos hospitais da amostra na fronteira de eficiência. Quanto ao número de leitos, independentemente da região, é maior a frequência de hospitais eficientes que possuem acima de 30 , e entre hospitais ineficientes a maior concentração 
Tabela 1 Hospitais eficientes e ineficientes por número de leitos, região e estado

\begin{tabular}{|c|c|c|c|c|c|c|c|c|c|c|c|c|}
\hline \multirow[t]{2}{*}{ Região/Estado } & \multicolumn{12}{|c|}{ 1-Eficiente } \\
\hline & & 0 a 19 & & 20 a 29 & & 30 a 39 & & 40 a 49 & Aci & na de 50 & & Total 1 \\
\hline Centro-0este & 8 & $6,15 \%$ & 19 & $14,62 \%$ & 17 & $13,08 \%$ & 1 & $0,77 \%$ & 9 & $6,92 \%$ & 54 & $41,54 \%$ \\
\hline Goiás & 3 & $3,70 \%$ & 7 & $8,64 \%$ & 8 & $9,88 \%$ & 1 & $1,23 \%$ & 7 & $8,64 \%$ & 26 & $32,10 \%$ \\
\hline Mato Grosso & 2 & $10,53 \%$ & 5 & $26,32 \%$ & 5 & $26,32 \%$ & & $0,00 \%$ & 1 & $5,26 \%$ & 13 & $68,42 \%$ \\
\hline $\begin{array}{l}\text { Mato Grosso } \\
\text { do Sul }\end{array}$ & 3 & $10,00 \%$ & 7 & $23,33 \%$ & 4 & $13,33 \%$ & & $0,00 \%$ & 1 & $3,33 \%$ & 15 & $50,00 \%$ \\
\hline Nordeste & 18 & $5,73 \%$ & 35 & $11,15 \%$ & 44 & $14,01 \%$ & 31 & $9,87 \%$ & 24 & $7,64 \%$ & 152 & $48,41 \%$ \\
\hline Alagoas & 1 & $4,76 \%$ & & $0,00 \%$ & 4 & $19,05 \%$ & 5 & $23,81 \%$ & 2 & $9,52 \%$ & 12 & $57,14 \%$ \\
\hline Bahia & 3 & $3,09 \%$ & 8 & $8,25 \%$ & 15 & $15,46 \%$ & 11 & $11,34 \%$ & 4 & $4,12 \%$ & 41 & $42,27 \%$ \\
\hline Ceará & 7 & $18,92 \%$ & 7 & $18,92 \%$ & 3 & $8,11 \%$ & 4 & $10,81 \%$ & 3 & $8,11 \%$ & 24 & $64,86 \%$ \\
\hline Maranhão & 3 & $6,82 \%$ & 13 & $29,55 \%$ & 6 & $13,64 \%$ & 3 & $6,82 \%$ & 1 & $2,27 \%$ & 26 & $59,09 \%$ \\
\hline Paraíba & & $0,00 \%$ & & $0,00 \%$ & 2 & $18,18 \%$ & 1 & $9,09 \%$ & 3 & $27,27 \%$ & 6 & $54,55 \%$ \\
\hline Pernambuco & 1 & $1,59 \%$ & & $0,00 \%$ & 6 & $9,52 \%$ & 2 & $3,17 \%$ & 3 & $4,76 \%$ & 12 & $19,05 \%$ \\
\hline Piauí & 2 & $9,52 \%$ & 1 & $4,76 \%$ & 6 & $28,57 \%$ & 2 & $9,52 \%$ & 5 & $23,81 \%$ & 16 & $76,19 \%$ \\
\hline $\begin{array}{l}\text { Rio Grande } \\
\text { do Norte }\end{array}$ & 1 & $5,88 \%$ & 5 & $29,41 \%$ & 1 & $5,88 \%$ & 3 & $17,65 \%$ & 2 & $11,76 \%$ & 12 & $70,59 \%$ \\
\hline Sergipe & & $0,00 \%$ & 1 & $33,33 \%$ & 1 & $33,33 \%$ & & $0,00 \%$ & 1 & $33,33 \%$ & 3 & $100,00 \%$ \\
\hline Norte & 4 & $7,14 \%$ & 13 & $23,21 \%$ & 7 & $12,50 \%$ & 10 & $17,86 \%$ & 1 & $1,79 \%$ & 35 & $62,50 \%$ \\
\hline Acre & 1 & $11,11 \%$ & 2 & $22,22 \%$ & 1 & $11,11 \%$ & 1 & $11,11 \%$ & & $0,00 \%$ & 5 & $55,56 \%$ \\
\hline Amapá & & $0,00 \%$ & 1 & $50,00 \%$ & & $0,00 \%$ & 1 & $50,00 \%$ & & $0,00 \%$ & & $100,00 \%$ \\
\hline Amazonas & 1 & $5,88 \%$ & 3 & $17,65 \%$ & 1 & $5,88 \%$ & 3 & $17,65 \%$ & & $0,00 \%$ & 8 & $47,06 \%$ \\
\hline Pará & 2 & $10,00 \%$ & 1 & $5,00 \%$ & 4 & $20,00 \%$ & 4 & $20,00 \%$ & 1 & $5,00 \%$ & 12 & $60,00 \%$ \\
\hline Rondônia & & $0,00 \%$ & 2 & $50,00 \%$ & 1 & $25,00 \%$ & 1 & $25,00 \%$ & & $0,00 \%$ & 4 & $100,00 \%$ \\
\hline Tocantins & & $0,00 \%$ & 4 & $100,00 \%$ & & $0,00 \%$ & & $0,00 \%$ & & $0,00 \%$ & 4 & $100,00 \%$ \\
\hline Sudeste & 3 & $4,11 \%$ & 8 & $10,96 \%$ & 14 & $19,18 \%$ & 9 & $12,33 \%$ & 7 & $9,59 \%$ & 41 & $56,16 \%$ \\
\hline Espírito Santo & 1 & $6,67 \%$ & 4 & $26,67 \%$ & 5 & $33,33 \%$ & 3 & $20,00 \%$ & 1 & $6,67 \%$ & 14 & $93,33 \%$ \\
\hline Minas Gerais & 1 & $2,86 \%$ & & $0,00 \%$ & 6 & $17,14 \%$ & 4 & $11,43 \%$ & 3 & $8,57 \%$ & 14 & $40,00 \%$ \\
\hline Rio de Janeiro & & $0,00 \%$ & 1 & $20,00 \%$ & 2 & $40,00 \%$ & 1 & $20,00 \%$ & 1 & $20,00 \%$ & 5 & $100,00 \%$ \\
\hline São Paulo & 1 & $5,56 \%$ & 3 & $16,67 \%$ & 1 & $5,56 \%$ & 1 & $5,56 \%$ & 2 & $11,11 \%$ & 8 & $44,44 \%$ \\
\hline Sul & 5 & $3,88 \%$ & 8 & $6,20 \%$ & 17 & $13,18 \%$ & 7 & $5,43 \%$ & 10 & $7,75 \%$ & 47 & $36,43 \%$ \\
\hline Paraná & 5 & $4,95 \%$ & 7 & $6,93 \%$ & 8 & $7,92 \%$ & 6 & $5,94 \%$ & 5 & $4,95 \%$ & 31 & $30,69 \%$ \\
\hline Rio Grande do Sul & & $0,00 \%$ & & $0,00 \%$ & 3 & $75,00 \%$ & & $0,00 \%$ & 1 & $25,00 \%$ & 4 & $100,00 \%$ \\
\hline Santa Catarina & & $0,00 \%$ & 1 & $4,17 \%$ & 6 & $25,00 \%$ & 1 & $4,17 \%$ & 4 & $16,67 \%$ & 12 & $50,00 \%$ \\
\hline Total & 38 & $5,41 \%$ & 83 & $11,82 \%$ & 99 & $14,10 \%$ & 58 & $8,26 \%$ & 51 & $7,26 \%$ & 329 & $46,87 \%$ \\
\hline
\end{tabular}




\begin{tabular}{|c|c|c|c|c|c|c|c|c|c|c|c|c|c|}
\hline & \multicolumn{11}{|c|}{2 - Ineficiente } & \multirow{2}{*}{\multicolumn{2}{|c|}{ Total gera }} \\
\hline & 0 a 19 & & 20 a 29 & & 30 a 39 & & 40 a 49 & \multicolumn{2}{|c|}{ Acima de 50} & \multicolumn{2}{|r|}{ Total 2} & & \\
\hline 34 & $26,15 \%$ & 23 & $17,69 \%$ & 16 & $12,31 \%$ & 2 & $1,54 \%$ & 1 & $0,77 \%$ & 76 & $58,46 \%$ & 130 & $100,00 \%$ \\
\hline 28 & $34,57 \%$ & 14 & $17,28 \%$ & 10 & $12,35 \%$ & 2 & $2,47 \%$ & 1 & $1,23 \%$ & 55 & $67,90 \%$ & 81 & $100,00 \%$ \\
\hline 2 & $10,53 \%$ & 2 & $10,53 \%$ & 2 & $10,53 \%$ & & $0,00 \%$ & & $0,00 \%$ & 6 & $31,58 \%$ & 19 & $100,00 \%$ \\
\hline 4 & $13,33 \%$ & 7 & $23,33 \%$ & 4 & $13,33 \%$ & & $0,00 \%$ & & $0,00 \%$ & 15 & $50,00 \%$ & 30 & $100,00 \%$ \\
\hline 45 & $14,33 \%$ & 66 & $21,02 \%$ & 30 & $9,55 \%$ & 17 & $5,41 \%$ & 4 & $1,27 \%$ & 162 & $51,59 \%$ & 314 & $100,00 \%$ \\
\hline 1 & $4,76 \%$ & 2 & $9,52 \%$ & 2 & $9,52 \%$ & 3 & $14,29 \%$ & 1 & $4,76 \%$ & 9 & $42,86 \%$ & 21 & $100,00 \%$ \\
\hline 13 & $13,40 \%$ & 23 & $23,71 \%$ & 12 & $12,37 \%$ & 5 & $5,15 \%$ & 3 & $3,09 \%$ & 56 & $57,73 \%$ & 97 & $100,00 \%$ \\
\hline 5 & $13,51 \%$ & 4 & $10,81 \%$ & 1 & $2,70 \%$ & 3 & $8,11 \%$ & & $0,00 \%$ & 13 & $35,14 \%$ & 37 & $100,00 \%$ \\
\hline 1 & $2,27 \%$ & 10 & $22,73 \%$ & 3 & $6,82 \%$ & 4 & $9,09 \%$ & & $0,00 \%$ & 18 & $40,91 \%$ & 44 & $100,00 \%$ \\
\hline 4 & $36,36 \%$ & & $0,00 \%$ & 1 & $9,09 \%$ & & $0,00 \%$ & & $0,00 \%$ & 5 & $45,45 \%$ & 11 & $100,00 \%$ \\
\hline 19 & $30,16 \%$ & 21 & $33,33 \%$ & 9 & $14,29 \%$ & 2 & $3,17 \%$ & & $0,00 \%$ & 51 & $80,95 \%$ & 63 & $100,00 \%$ \\
\hline 1 & $4,76 \%$ & 3 & $14,29 \%$ & 1 & $4,76 \%$ & & $0,00 \%$ & & $0,00 \%$ & 5 & $23,81 \%$ & 21 & $100,00 \%$ \\
\hline 1 & $5,88 \%$ & 3 & $17,65 \%$ & 1 & $5,88 \%$ & & $0,00 \%$ & & $0,00 \%$ & 5 & $29,41 \%$ & 17 & $100,00 \%$ \\
\hline & $0,00 \%$ & & $0,00 \%$ & & $0,00 \%$ & & $0,00 \%$ & & $0,00 \%$ & & $0,00 \%$ & 3 & $100,00 \%$ \\
\hline 8 & $14,29 \%$ & 11 & $19,64 \%$ & & $0,00 \%$ & 2 & $3,57 \%$ & & $0,00 \%$ & 21 & $37,50 \%$ & 56 & $100,00 \%$ \\
\hline 4 & $44,44 \%$ & & $0,00 \%$ & & $0,00 \%$ & & $0,00 \%$ & & $0,00 \%$ & 4 & $44,44 \%$ & 9 & $100,00 \%$ \\
\hline & $0,00 \%$ & & $0,00 \%$ & & $0,00 \%$ & & $0,00 \%$ & & $0,00 \%$ & & $0,00 \%$ & 2 & $100,00 \%$ \\
\hline 2 & $11,76 \%$ & 6 & $35,29 \%$ & & $0,00 \%$ & 1 & $5,88 \%$ & & $0,00 \%$ & 9 & $52,94 \%$ & 17 & $100,00 \%$ \\
\hline 2 & $10,00 \%$ & 5 & $25,00 \%$ & & $0,00 \%$ & 1 & $5,00 \%$ & & $0,00 \%$ & 8 & $40,00 \%$ & 20 & $100,00 \%$ \\
\hline & $0,00 \%$ & & $0,00 \%$ & & $0,00 \%$ & & $0,00 \%$ & & $0,00 \%$ & & $0,00 \%$ & 4 & $100,00 \%$ \\
\hline & $0,00 \%$ & & $0,00 \%$ & & $0,00 \%$ & & $0,00 \%$ & & $0,00 \%$ & & $0,00 \%$ & 4 & $100,00 \%$ \\
\hline 6 & $8,22 \%$ & 9 & $12,33 \%$ & 13 & $17,81 \%$ & 3 & $4,11 \%$ & 1 & $1,37 \%$ & 32 & $43,84 \%$ & 73 & $100,00 \%$ \\
\hline & $0,00 \%$ & 1 & $6,67 \%$ & & $0,00 \%$ & & $0,00 \%$ & & $0,00 \%$ & 1 & $6,67 \%$ & 15 & $100,00 \%$ \\
\hline 4 & $11,43 \%$ & 7 & $20,00 \%$ & 8 & $22,86 \%$ & 1 & $2,86 \%$ & 1 & $2,86 \%$ & 21 & $60,00 \%$ & 35 & $100,00 \%$ \\
\hline & $0,00 \%$ & & $0,00 \%$ & & $0,00 \%$ & & $0,00 \%$ & & $0,00 \%$ & & $0,00 \%$ & 5 & $100,00 \%$ \\
\hline 2 & $11,11 \%$ & 1 & $5,56 \%$ & 5 & $27,78 \%$ & 2 & $11,11 \%$ & & $0,00 \%$ & 10 & $55,56 \%$ & 18 & $100,00 \%$ \\
\hline 13 & $10,08 \%$ & 35 & $27,13 \%$ & 24 & $18,60 \%$ & 7 & $5,43 \%$ & 3 & $2,33 \%$ & 82 & $63,57 \%$ & 129 & $100,00 \%$ \\
\hline 13 & $12,87 \%$ & 31 & $30,69 \%$ & 19 & $18,81 \%$ & 4 & $3,96 \%$ & 3 & $2,97 \%$ & 70 & $69,31 \%$ & 101 & $100,00 \%$ \\
\hline & $0,00 \%$ & & $0,00 \%$ & & $0,00 \%$ & & $0,00 \%$ & & $0,00 \%$ & & $0,00 \%$ & 4 & $100,00 \%$ \\
\hline & $0,00 \%$ & 4 & $16,67 \%$ & 5 & $20,83 \%$ & 3 & $12,50 \%$ & & $0,00 \%$ & 12 & $50,00 \%$ & 24 & $100,00 \%$ \\
\hline 106 & $15,10 \%$ & 144 & $20,51 \%$ & 83 & $11,82 \%$ & 31 & $4,42 \%$ & 9 & $1,28 \%$ & 373 & $53,13 \%$ & 702 & $100,00 \%$ \\
\hline
\end{tabular}

Fonte: Elaboração própria. 
Tabela 2 Distribuição dos hospitais eficientes e ineficientes por natureza jurídica,

\begin{tabular}{|c|c|c|c|c|c|c|c|c|c|c|c|c|}
\hline \multirow{3}{*}{$\begin{array}{l}\text { Região/Estado } \\
\text { Centro-Oeste }\end{array}$} & \multicolumn{12}{|c|}{1 - Eficiente } \\
\hline & \multicolumn{2}{|c|}{$\begin{array}{r}\text { Privado com } \\
\text { fins lucrativos }\end{array}$} & \multicolumn{2}{|c|}{$\begin{array}{r}\text { Privado sem } \\
\text { fins lucrativos }\end{array}$} & \multirow{2}{*}{\multicolumn{2}{|c|}{$\begin{array}{r}\begin{array}{r}\text { Público } \\
\text { estadual }\end{array} \\
0,00 \%\end{array}$}} & \multirow{2}{*}{\multicolumn{2}{|c|}{$\begin{array}{r}\begin{array}{r}\text { Público } \\
\text { federal }\end{array} \\
0,00 \%\end{array}$}} & \multicolumn{2}{|r|}{$\begin{array}{r}\text { Público } \\
\text { municipal }\end{array}$} & \multicolumn{2}{|r|}{ Total } \\
\hline & 8 & $6,15 \%$ & 10 & $7,69 \%$ & & & & & 36 & $27,69 \%$ & 54 & $4 \quad 41,54 \%$ \\
\hline Goiás & 5 & $6,17 \%$ & 3 & $3,70 \%$ & & $0,00 \%$ & & $0,00 \%$ & 18 & $22,22 \%$ & 26 & $6 \quad 32,10 \%$ \\
\hline Mato Grosso & 3 & $15,79 \%$ & 2 & $10,53 \%$ & & $0,00 \%$ & & $0,00 \%$ & 8 & $42,11 \%$ & 13 & $3 \quad 68,42 \%$ \\
\hline $\begin{array}{l}\text { Mato Grosso } \\
\text { do Sul }\end{array}$ & & $0,00 \%$ & 5 & $16,67 \%$ & & $0,00 \%$ & & $0,00 \%$ & 10 & $33,33 \%$ & 15 & $5 \quad 50,00 \%$ \\
\hline Nordeste & 4 & $1,27 \%$ & 11 & $3,50 \%$ & 26 & $8,28 \%$ & 2 & $0,64 \%$ & 109 & $34,71 \%$ & 152 & $248,41 \%$ \\
\hline Alagoas & 1 & $4,76 \%$ & & $0,00 \%$ & 3 & $14,29 \%$ & & $0,00 \%$ & 8 & $38,10 \%$ & 12 & $57,14 \%$ \\
\hline Bahia & 2 & $2,06 \%$ & 6 & $6,19 \%$ & 5 & $5,15 \%$ & & $0,00 \%$ & 28 & $28,87 \%$ & 41 & $142,27 \%$ \\
\hline Ceará & & $0,00 \%$ & & $0,00 \%$ & & $0,00 \%$ & & $0,00 \%$ & 24 & $64,86 \%$ & 24 & $4 \quad 64,86 \%$ \\
\hline Maranhão & & $0,00 \%$ & & $0,00 \%$ & 4 & $9,09 \%$ & 1 & $2,27 \%$ & 21 & $47,73 \%$ & 26 & $6 \quad 59,09 \%$ \\
\hline Paraíba & 1 & $9,09 \%$ & & $0,00 \%$ & 2 & $18,18 \%$ & & $0,00 \%$ & 3 & $27,27 \%$ & 6 & $6 \quad 54,55 \%$ \\
\hline Pernambuco & & $0,00 \%$ & 2 & $3,17 \%$ & 2 & $3,17 \%$ & & $0,00 \%$ & 8 & $12,70 \%$ & 12 & $2 \quad 19,05 \%$ \\
\hline Piauí & & $0,00 \%$ & 1 & $4,76 \%$ & 5 & $23,81 \%$ & & $0,00 \%$ & 10 & $47,62 \%$ & 16 & $6 \quad 76,19 \%$ \\
\hline $\begin{array}{l}\text { Rio Grande } \\
\text { do Norte }\end{array}$ & & $0,00 \%$ & 1 & $5,88 \%$ & 3 & $17,65 \%$ & 1 & $5,88 \%$ & 7 & $41,18 \%$ & 12 & $270,59 \%$ \\
\hline Sergipe & & $0,00 \%$ & 1 & $33,33 \%$ & 2 & $66,67 \%$ & & $0,00 \%$ & & $0,00 \%$ & & $3100,00 \%$ \\
\hline Norte & 2 & $3,57 \%$ & 1 & $1,79 \%$ & 14 & $25,00 \%$ & 1 & $1,79 \%$ & 17 & $30,36 \%$ & 35 & $5 \quad 62,50 \%$ \\
\hline Acre & & $0,00 \%$ & & $0,00 \%$ & 5 & $55,56 \%$ & & $0,00 \%$ & & $0,00 \%$ & & $5 \quad 55,56 \%$ \\
\hline Amapá & & $0,00 \%$ & & $0,00 \%$ & 1 & $50,00 \%$ & & $0,00 \%$ & 1 & $50,00 \%$ & & $2100,00 \%$ \\
\hline Amazonas & 1 & $5,88 \%$ & & $0,00 \%$ & 5 & $29,41 \%$ & & $0,00 \%$ & 2 & $11,76 \%$ & 8 & $8 \quad 47,06 \%$ \\
\hline Pará & 1 & $5,00 \%$ & 1 & $5,00 \%$ & 2 & $10,00 \%$ & 1 & $5,00 \%$ & 7 & $35,00 \%$ & 12 & $260,00 \%$ \\
\hline Rondônia & & $0,00 \%$ & & $0,00 \%$ & 1 & $25,00 \%$ & & $0,00 \%$ & 3 & $75,00 \%$ & & $4100,00 \%$ \\
\hline Tocantins & & $0,00 \%$ & & $0,00 \%$ & & $0,00 \%$ & & $0,00 \%$ & 4 & $100,00 \%$ & & $4 \quad 100,00 \%$ \\
\hline Sudeste & 3 & $4,11 \%$ & 22 & $30,14 \%$ & 2 & $2,74 \%$ & & $0,00 \%$ & 14 & $19,18 \%$ & 41 & $156,16 \%$ \\
\hline Espírito Santo & 1 & $6,67 \%$ & 7 & $46,67 \%$ & 2 & $13,33 \%$ & & $0,00 \%$ & 4 & $26,67 \%$ & 14 & $4 \quad 93,33 \%$ \\
\hline Minas Gerais & 2 & $5,71 \%$ & 11 & $31,43 \%$ & & $0,00 \%$ & & $0,00 \%$ & 1 & $2,86 \%$ & 14 & $4 \quad 40,00 \%$ \\
\hline Rio de Janeiro & & $0,00 \%$ & 1 & $20,00 \%$ & & $0,00 \%$ & & $0,00 \%$ & 4 & $80,00 \%$ & & $5100,00 \%$ \\
\hline São Paulo & & $0,00 \%$ & 3 & $16,67 \%$ & & $0,00 \%$ & & $0,00 \%$ & 5 & $27,78 \%$ & 8 & $8 \quad 44,44 \%$ \\
\hline Sul & 7 & $5,43 \%$ & 16 & $12,40 \%$ & & $0,00 \%$ & 2 & $1,55 \%$ & 22 & $17,05 \%$ & 47 & $7 \quad 36,43 \%$ \\
\hline Paraná & 5 & $4,95 \%$ & 6 & $5,94 \%$ & & $0,00 \%$ & 2 & $1,98 \%$ & 18 & $17,82 \%$ & 31 & $130,69 \%$ \\
\hline Rio Grande do Sul & & $0,00 \%$ & 3 & $75,00 \%$ & & $0,00 \%$ & & $0,00 \%$ & 1 & $25,00 \%$ & & $4 \quad 100,00 \%$ \\
\hline Santa Catarina & 2 & $8,33 \%$ & 7 & $29,17 \%$ & & $0,00 \%$ & & $0,00 \%$ & 3 & $12,50 \%$ & 12 & $250,00 \%$ \\
\hline Total & 24 & $3,42 \%$ & 60 & $8,55 \%$ & 42 & $5,98 \%$ & 5 & $0,71 \%$ & 198 & $28,21 \%$ & 329 & 9 $46,87 \%$ \\
\hline
\end{tabular}


região e estado

\begin{tabular}{|c|c|c|c|c|c|c|c|c|c|c|c|c|c|}
\hline & & & & & & & & & & $2-\ln$ & leficiente & & Total geral \\
\hline $\begin{array}{r}\text { Priv } \\
\text { fins It }\end{array}$ & $\begin{array}{l}\text { do com } \\
\text { crativos }\end{array}$ & & $\begin{array}{l}\text { lado sem } \\
\text { Icrativos }\end{array}$ & & $\begin{array}{l}\text { Público } \\
\text { estadual }\end{array}$ & & $\begin{array}{l}\text { Público } \\
\text { federal }\end{array}$ & & $\begin{array}{l}\text { Público } \\
\text { nunicipal }\end{array}$ & & Total 2 & & \\
\hline 4 & $3,08 \%$ & 9 & $6,92 \%$ & 1 & $0,77 \%$ & 1 & $0,77 \%$ & 61 & $46,92 \%$ & 76 & $58,46 \%$ & 130 & $100,00 \%$ \\
\hline 4 & $4,94 \%$ & 1 & $1,23 \%$ & 1 & $1,23 \%$ & 1 & $1,23 \%$ & 48 & $59,26 \%$ & 55 & $67,90 \%$ & 81 & $100,00 \%$ \\
\hline & $0,00 \%$ & 1 & $5,26 \%$ & & $0,00 \%$ & & $0,00 \%$ & 5 & $26,32 \%$ & 6 & $31,58 \%$ & 19 & $100,00 \%$ \\
\hline & $0,00 \%$ & 7 & $23,33 \%$ & & $0,00 \%$ & & $0,00 \%$ & 8 & $26,67 \%$ & 15 & $50,00 \%$ & 30 & $100,00 \%$ \\
\hline 4 & $1,27 \%$ & 5 & $1,59 \%$ & 6 & $1,91 \%$ & 2 & $0,64 \%$ & 145 & $46,18 \%$ & 162 & $51,59 \%$ & 314 & $100,00 \%$ \\
\hline & $0,00 \%$ & & $0,00 \%$ & & $0,00 \%$ & & $0,00 \%$ & 9 & $42,86 \%$ & 9 & $42,86 \%$ & 21 & $100,00 \%$ \\
\hline 2 & $2,06 \%$ & 1 & $1,03 \%$ & 3 & $3,09 \%$ & 2 & $2,06 \%$ & 48 & $49,48 \%$ & 56 & $57,73 \%$ & 97 & $100,00 \%$ \\
\hline & $0,00 \%$ & 2 & $5,41 \%$ & & $0,00 \%$ & & $0,00 \%$ & 11 & $29,73 \%$ & 13 & $35,14 \%$ & 37 & $100,00 \%$ \\
\hline 1 & $2,27 \%$ & & $0,00 \%$ & & $0,00 \%$ & & $0,00 \%$ & 17 & $38,64 \%$ & 18 & $40,91 \%$ & 44 & $100,00 \%$ \\
\hline & $0,00 \%$ & & $0,00 \%$ & & $0,00 \%$ & & $0,00 \%$ & 5 & $45,45 \%$ & 5 & $45,45 \%$ & 11 & $100,00 \%$ \\
\hline & $0,00 \%$ & & $0,00 \%$ & & $0,00 \%$ & & $0,00 \%$ & 51 & $80,95 \%$ & 51 & $80,95 \%$ & 63 & $100,00 \%$ \\
\hline 1 & $4,76 \%$ & & $0,00 \%$ & 2 & $9,52 \%$ & & $0,00 \%$ & 2 & $9,52 \%$ & 5 & 23,81\% & 21 & $100,00 \%$ \\
\hline & $0,00 \%$ & 2 & $11,76 \%$ & 1 & $5,88 \%$ & & $0,00 \%$ & 2 & $11,76 \%$ & 5 & $29,41 \%$ & 17 & $100,00 \%$ \\
\hline & $0,00 \%$ & & $0,00 \%$ & & $0,00 \%$ & & $0,00 \%$ & & $0,00 \%$ & & $0,00 \%$ & 3 & $100,00 \%$ \\
\hline & $0,00 \%$ & & $0,00 \%$ & 13 & $23,21 \%$ & 1 & $1,79 \%$ & 7 & $12,50 \%$ & 21 & $37,50 \%$ & 56 & $100,00 \%$ \\
\hline & $0,00 \%$ & & $0,00 \%$ & 4 & $44,44 \%$ & & $0,00 \%$ & & $0,00 \%$ & 4 & $44,44 \%$ & 9 & $100,00 \%$ \\
\hline & $0,00 \%$ & & $0,00 \%$ & & $0,00 \%$ & & $0,00 \%$ & & $0,00 \%$ & & $0,00 \%$ & 2 & $100,00 \%$ \\
\hline & $0,00 \%$ & & $0,00 \%$ & 9 & $52,94 \%$ & & $0,00 \%$ & & $0,00 \%$ & 9 & $52,94 \%$ & 17 & $100,00 \%$ \\
\hline & $0,00 \%$ & & $0,00 \%$ & & $0,00 \%$ & 1 & $5,00 \%$ & 7 & $35,00 \%$ & 8 & $40,00 \%$ & 20 & $100,00 \%$ \\
\hline & $0,00 \%$ & & $0,00 \%$ & & $0,00 \%$ & & $0,00 \%$ & & $0,00 \%$ & & $0,00 \%$ & 4 & $100,00 \%$ \\
\hline & $0,00 \%$ & & $0,00 \%$ & & $0,00 \%$ & & $0,00 \%$ & & $0,00 \%$ & & $0,00 \%$ & 4 & $100,00 \%$ \\
\hline & $0,00 \%$ & 18 & $24,66 \%$ & & $0,00 \%$ & & $0,00 \%$ & 14 & $19,18 \%$ & 32 & $43,84 \%$ & 73 & $100,00 \%$ \\
\hline & $0,00 \%$ & & $0,00 \%$ & & $0,00 \%$ & & $0,00 \%$ & 1 & $6,67 \%$ & 1 & $6,67 \%$ & 15 & $100,00 \%$ \\
\hline & $0,00 \%$ & 14 & $40,00 \%$ & & $0,00 \%$ & & $0,00 \%$ & 7 & $20,00 \%$ & 21 & $60,00 \%$ & 35 & $100,00 \%$ \\
\hline & $0,00 \%$ & & $0,00 \%$ & & $0,00 \%$ & & $0,00 \%$ & & $0,00 \%$ & & $0,00 \%$ & 5 & $100,00 \%$ \\
\hline & $0,00 \%$ & 4 & $22,22 \%$ & & $0,00 \%$ & & $0,00 \%$ & 6 & $33,33 \%$ & 10 & $55,56 \%$ & 18 & $100,00 \%$ \\
\hline 12 & $9,30 \%$ & 18 & $13,95 \%$ & 4 & $3,10 \%$ & & $0,00 \%$ & 48 & $37,21 \%$ & 82 & $63,57 \%$ & 129 & $100,00 \%$ \\
\hline 12 & $11,88 \%$ & 12 & $11,88 \%$ & 3 & $2,97 \%$ & & $0,00 \%$ & 43 & $42,57 \%$ & 70 & $69,31 \%$ & 101 & $100,00 \%$ \\
\hline & $0,00 \%$ & & $0,00 \%$ & & $0,00 \%$ & & $0,00 \%$ & & $0,00 \%$ & & $0,00 \%$ & 4 & $100,00 \%$ \\
\hline & $0,00 \%$ & 6 & $25,00 \%$ & 1 & $4,17 \%$ & & $0,00 \%$ & 5 & $20,83 \%$ & 12 & $50,00 \%$ & 24 & $100,00 \%$ \\
\hline 20 & $2,85 \%$ & 50 & $7,12 \%$ & 24 & $3,42 \%$ & 4 & $0,57 \%$ & 275 & $39,17 \%$ & 373 & $53,13 \%$ & 702 & $100,00 \%$ \\
\hline
\end{tabular}

Fonte: Elaboração própria. 
está no intervalo entre 20 e 29 (20,51\%) e 0 a 19 leitos (15,10\%). Importante destacar que entre os hospitais acima de 50 leitos, quase a totalidade está na fronteira de eficiência. As diferenças desse padrão ocorreram: a) na região Norte com percentual maior de hospitais eficientes, comparativamente aos ineficientes, no intervalo de 20 a 29 leitos (23,21\%), e b) na região Sul com percentual maior de hospitais ineficientes no intervalo de 30 a 39 leitos e percentual equivalente para aqueles com 40 a 49 leitos.

O resultado do modelo de eficiência quanto à natureza jurídica evidencia a maior parte dos hospitais de gestão pública municipal fora da fronteira de eficiência (Tabela 2). Em âmbito nacional, nos hospitais privados com e sem fins lucrativos, públicos estaduais e federais, a frequência maior é de hospitais eficientes. As configurações que diferenciam dessa referência em nível regional são: a) no Nordeste é equivalente a frequência de hospitais eficientes e ineficientes classificados como privados sem fins lucrativos; b) no Norte é maior a frequência de hospitais públicos municipais na fronteira de eficiência; c) no Sudeste é equivalente a distribuição entre os hospitais eficientes e ineficientes na gestão pública municipal, e d) no Sul é menor a frequência de hospitais eficientes entre os hospitais privados com e sem lucrativos e também públicos estaduais.

Os hospitais eficientes possuem estrutura, processos e resultados com padrão superior àqueles não eficientes em âmbito nacional (Tabelas 3 a 5). A comparação, entre os hospitais eficientes e ineficientes, na dimensão estrutura evidencia:

a) mesmo padrão entre as práticas dos hospitais eficientes e ineficientes na região Sudeste para todas as categorias de análise;

b) na região Norte somente a categoria políticas de recursos humanos possui padrão superior dos hospitais eficientes em relação aos ineficientes;

c) nas regiões Centro-Oeste e Nordeste somente as práticas referentes à categoria força de trabalho possuem padrão próximo entre os hospitais eficientes e ineficientes. Para as demais categorias as práticas dos hospitais eficientes são superiores;

d) na região Sul as condições referentes às salas de atendimento e urgência e emergência são equivalentes entre os hospitais eficientes e ineficientes. 
Tabela 3 Média dos inputs da dimensão de estrutura por região

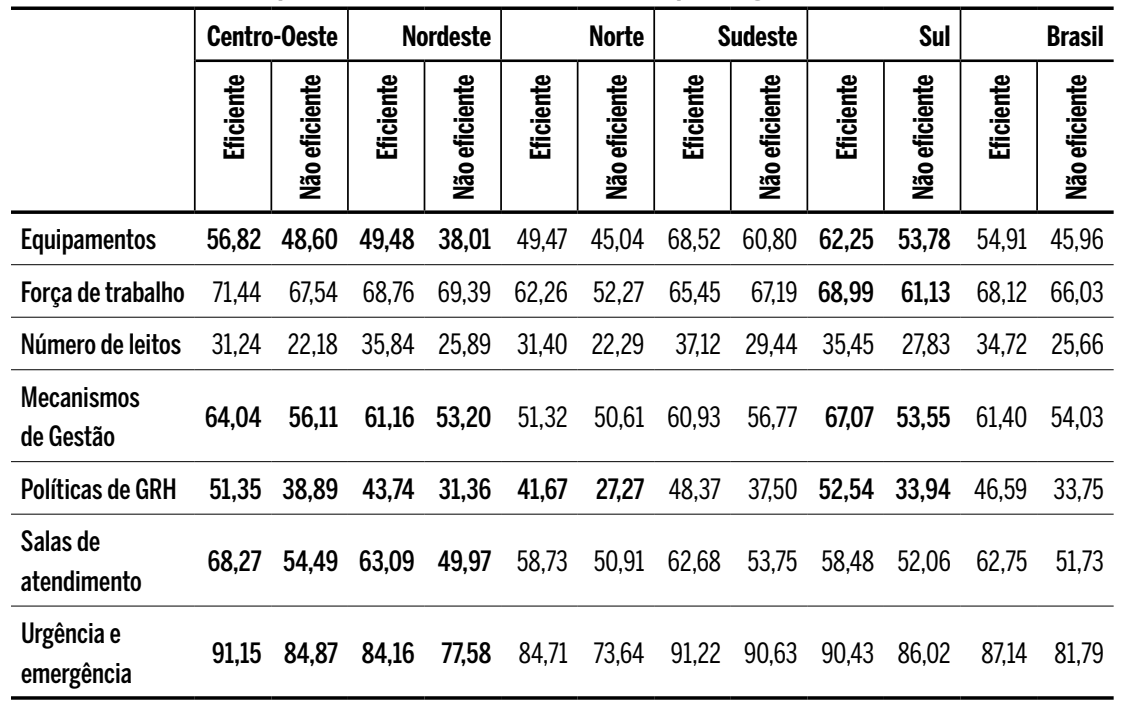

Fonte: Elaboração própria.

Na dimensão de processo a comparação entre hospitais eficientes e ineficientes demonstra:

a) mesmo padrão entre as práticas dos hospitais eficientes e ineficientes na região Norte para todas as categorias;

b) na região Sudeste somente as categorias serviços e monitoramento dos indicadores assistenciais indicam padrão superior dos hospitais eficientes em relação aos ineficientes;

c) nas regiões Centro-Oeste e Sul somente a categoria regulação possui padrão próximo entre os hospitais eficientes e ineficientes. Para as demais categorias, as práticas dos hospitais eficientes são superiores;

d) na região Nordeste somente as práticas referentes aos dispositivos são equivalentes entre os hospitais eficientes e ineficientes. Para as demais categorias, as práticas dos hospitais eficientes são superiores. 
Tabela 4 Média dos inputs da dimensão de processo por região

\begin{tabular}{|c|c|c|c|c|c|c|c|c|c|c|c|c|}
\hline & \multicolumn{2}{|c|}{ Centro-0este } & \multicolumn{2}{|c|}{ Nordeste } & \multicolumn{2}{|r|}{ Norte } & \multicolumn{2}{|c|}{ Sudeste } & \multicolumn{2}{|r|}{ Sul } & \multicolumn{2}{|r|}{ Brasil } \\
\hline & 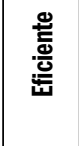 & 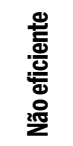 & 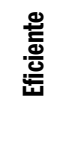 & 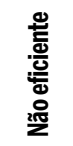 & 畩 & 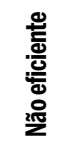 & 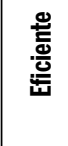 & 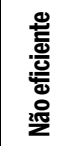 & 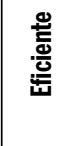 & 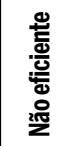 & 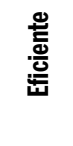 & 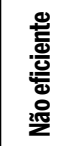 \\
\hline Dispositivos & 73,72 & 72,01 & 73,74 & 67,19 & 63,73 & 63,64 & 65,85 & 60,94 & 72,10 & 62,25 & 71,44 & 66,37 \\
\hline $\begin{array}{l}\text { Monitoramento } \\
\text { de indicadores } \\
\text { assistenciais }\end{array}$ & 74,27 & 51,74 & 61,22 & 41,76 & 55,46 & 52,60 & 78,92 & 52,68 & 81,36 & 49,66 & 67,85 & 47,08 \\
\hline $\begin{array}{l}\text { Processo } \\
\text { de trabalho }\end{array}$ & 71,71 & 78,80 & 72,85 & 65,92 & 70,87 & 68,60 & 78,90 & 79,83 & 82,69 & 75,90 & 75,78 & 70,62 \\
\hline Regulação & 70,34 & 72,15 & 70,10 & 63,67 & 62,11 & 67,42 & 51,22 & 48,44 & 58,23 & 56,97 & 65,19 & 62,88 \\
\hline Serviços & 77,44 & 69,15 & 76,15 & 68,47 & 73,92 & 66,06 & 80,49 & 68,13 & 78,26 & 65,30 & 76,98 & 67,75 \\
\hline $\begin{array}{l}\text { Serviços } \\
\text { especializados }\end{array}$ & 54,09 & 44,78 & 55,25 & 42,85 & 54,04 & 48,86 & 50,91 & 43,14 & 54,35 & 41,65 & 54,25 & 43,36 \\
\hline
\end{tabular}

Fonte: Elaboração própria.

Em relação às variáveis de resultado, de acordo com a Tabela 5:

a) a frequência de AIHs possui padrão superior entre os hospitais eficientes em todas as regiões;

b) o valor médio das AIHs é equivalente entre hospitais eficientes e ineficientes somente na região Norte. Para as demais regiões é superior entre os hospitais eficientes;

c) o total de procedimentos realizados é equivalente entre hospitais eficientes e ineficientes nas regiões Centro-Oeste e Sudeste.

Tabela 5 Média dos inputs da dimensão de resultados por região

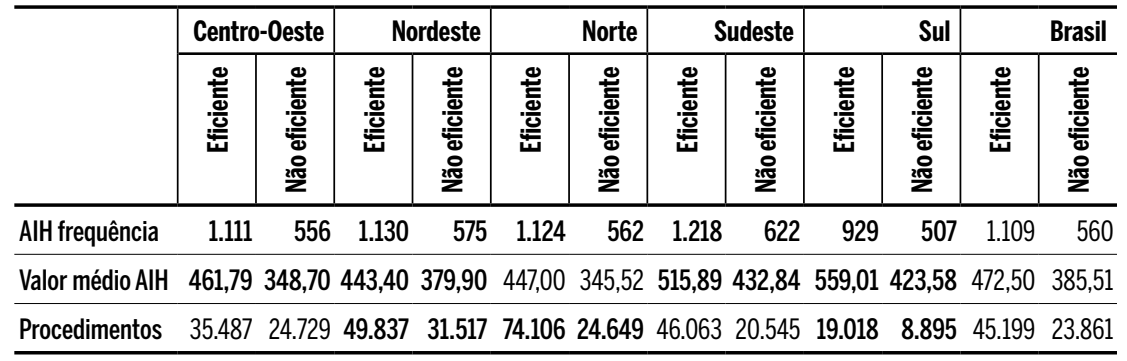

Fonte: Elaboração própria. 
A fronteira de eficiência é caracterizada por hospitais públicos de gestão municipal com quantitativo de leitos superior a 30 e com as práticas de estrutura, processo e resultados com padrão diferenciado em relação aos hospitais ineficientes. A partir do resultado do modelo de eficiência foi possível identificar as principais características dos hospitais que não atingiram a fronteira de eficiência quanto à distribuição nas regiões do país, número de leitos e natureza jurídica. Essa delimitação serve como referência para o detalhamento do grupo de hospitais ineficientes frente ao conteúdo relacionado com a melhoria da eficiência. A Tabela 6 apresenta a média de melhoria necessária para os inputs e outputs para cada agrupamento de variáveis por região.

A combinação das variáveis com maior potencial para melhoria da eficiência dos hospitais de pequeno porte é diferente em cada região do país. As variáveis que possuem maior score para alcance da fronteira de eficiência são:

a) Centro-Oeste: políticas de Recursos Humanos (RH), número de leitos e monitoramento de indicadores assistenciais;

b) Nordeste: monitoramento de indicadores assistenciais, políticas de $\mathrm{RH}$ e serviços especializados;

c) Norte: políticas de RH, número de leitos e salas de atendimento;

d) Sudeste: políticas de RH, número de leitos e monitoramento de indicadores assistenciais;

e) Sul: políticas de RH, serviços especializados e mecanismos de gestão. Com base no número de leitos a Tabela 7 demonstra que o percentual de incremento, para as todas as variáveis do modelo, é decrescente quanto maior o número de leitos e que a partir de 50 leitos não necessita aumento dessa variável para melhoria da eficiência dos hospitais, que comparativamente aos demais se encontram próximos da fronteira de eficiência.

No entanto, cada intervalo de leitos apresenta um arranjo específico das variáveis com potencial de melhoria. A variável do número de leitos somente tem efeito mais significativo para a eficiência entre os hospitais menores (0 a 19 leitos), demonstrando que a capacidade deveria ser o dobro em relação à disponibilidade atual de leitos. Ao mesmo tempo o incremento necessário para o total de procedimentos e a frequência de AIHs é superior a cinco vezes ao praticado na realidade atual dos hospitais ineficientes.

Com base na distribuição dos hospitais por região e a natureza jurídica, embora a caracterização geral evidencie maior frequência dos públicos municipais é importante destacar que esse padrão não é o mesmo para todas 


\begin{tabular}{|c|c|c|c|c|c|c|c|c|c|c|c|c|c|c|c|}
\hline & 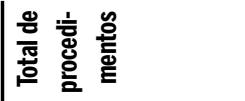 & $\left|\begin{array}{c}\infty \\
\infty \\
\sim \\
\sim\end{array}\right|$ & 突 & $\begin{array}{l}g \\
g \\
\infty \\
\infty\end{array}$ & 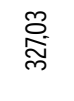 & 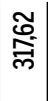 & $\left|\begin{array}{c}\stackrel{\infty}{\infty} \\
\underset{\sim}{\sim}\end{array}\right|$ & 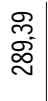 & 总 & $\mid$ & $\left|\begin{array}{l}q \\
0 \\
6 \\
6\end{array}\right|$ & $\mid$\begin{tabular}{l} 
足 \\
\multirow{2}{*}{}
\end{tabular} & 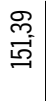 & 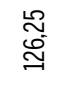 & 8 \\
\hline (1) & 존 흘 음 & 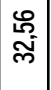 & $\begin{array}{l}\widetilde{0} \\
0 \\
\sigma \\
\sigma\end{array}$ & $\begin{array}{l}\text { 芯 } \\
\stackrel{0}{0}\end{array}$ & 兽 & 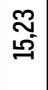 & 吕 & $\begin{array}{l}\vec{\sim} \\
\text { เ్ }\end{array}$ & 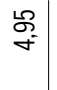 & $\begin{array}{l}8 \\
\stackrel{8}{\circ} \\
\infty\end{array}$ & $\mid \begin{array}{c}0 \\
0 \\
0 \\
0\end{array}$ & \begin{tabular}{|l}
$\vec{n}$ \\
$\stackrel{2}{N}$
\end{tabular} & 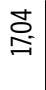 & 壱 & 8 \\
\hline - & 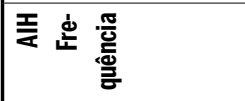 & 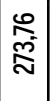 & 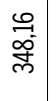 & 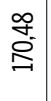 & $\underset{\sim}{\stackrel{\infty}{్}}$ & $\begin{array}{l}\bar{c} \\
\bar{e} \\
\tilde{m}\end{array}$ & \begin{tabular}{|l}
$\widetilde{\omega}$ \\
$\widetilde{\Omega}$ \\
$\stackrel{\sim}{\sim}$
\end{tabular} & 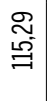 & 壳 & 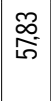 & 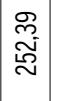 & $\begin{array}{l}\infty \\
0 \\
0 \\
m \\
m\end{array}$ & 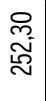 & 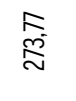 & 8 \\
\hline U. & 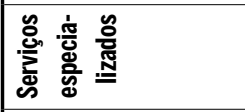 & 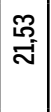 & $\begin{array}{l}8 \\
\stackrel{i}{0}\end{array}$ & $\begin{array}{l}\infty \\
0 \\
0\end{array}$ & $\begin{array}{l}\mathscr{\infty} \\
\stackrel{\sim}{*}\end{array}$ & $\begin{array}{l}\vec{m} \\
\vec{\nabla}\end{array}$ & $\begin{array}{l}\mathscr{P} \\
\tilde{\sigma}\end{array}$ & 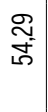 & 罸 & $\stackrel{\infty}{\stackrel{\infty}{\sim}}$ & $\begin{array}{c}0 \\
\stackrel{\infty}{\sim}\end{array}$ & 兑 & 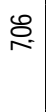 & $\begin{array}{l}0 \\
6 \\
6 \\
0\end{array}$ & 응 \\
\hline$\pi$ & 递 & $\begin{array}{c}\overrightarrow{3} \\
\stackrel{9}{9}\end{array}$ & $\underset{D}{\exists}$ & $\begin{array}{l}\mathscr{Q} \\
\stackrel{5}{\circ}\end{array}$ & 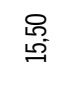 & $\begin{array}{l}\mathscr{\infty} \\
\stackrel{-}{\sim}\end{array}$ & $\underset{\underset{\sigma}{\tilde{\sigma}}}{\mathfrak{\Im}}$ & $\begin{array}{l}\ddot{B} \\
\stackrel{n}{n}\end{array}$ & 용 & $\begin{array}{l}\infty \\
\stackrel{2}{0} \\
\stackrel{0}{0}\end{array}$ & $\begin{array}{l}9 \\
\infty \\
\infty\end{array}$ & $\begin{array}{l}\mathscr{m} \\
\tilde{\sim} \\
\end{array}$ & $\begin{array}{l}\mathbb{\Xi} \\
\mathbb{6}\end{array}$ & $\stackrel{\mathscr{\infty}}{\stackrel{\infty}{=}}$ & 응 \\
\hline 등 & 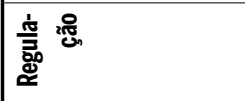 & $\underset{\sim}{\stackrel{0}{*}}$ & ָٓ & $\underset{f}{\stackrel{g}{f}}$ & 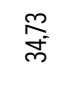 & $\begin{array}{l}\overrightarrow{\tilde{N}} \\
\text { }\end{array}$ & $\underset{\sigma}{\not}$ & $\underset{\mathbb{N}}{\tilde{\sim}}$ & $\stackrel{\widetilde{n}}{\sim}$ & $\stackrel{\sqrt{n}}{=}$ & $\underset{\sim}{\stackrel{i}{\sim}}$ & 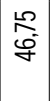 & $\stackrel{m}{\circ}$ & $\begin{array}{l}\vec{b} \\
\overrightarrow{\omega^{-}}\end{array}$ & 8 \\
\hline ద్ & 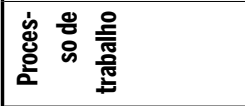 & $\begin{array}{l}\stackrel{8}{0} \\
\stackrel{0}{0}\end{array}$ & $\begin{array}{l}\dot{0} \\
\infty \\
\sim\end{array}$ & $\begin{array}{l}\stackrel{2}{0} \\
0 \\
0\end{array}$ & $\underset{\infty}{\mathbb{J}}$ & $\begin{array}{l}\text { 导 } \\
\text { G }\end{array}$ & $\frac{0}{\stackrel{0}{I}}$ & $\begin{array}{l}\infty \\
\stackrel{\infty}{=}\end{array}$ & 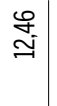 & 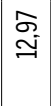 & $\begin{array}{c}\infty \\
\infty \\
\infty\end{array}$ & $\begin{array}{l}\stackrel{\otimes}{\sim} \\
\stackrel{-}{*}\end{array}$ & 농 & $\underset{m}{\infty}$ & 8 \\
\hline$\underline{\underline{v}}$ & 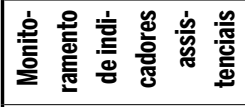 & 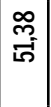 & $\begin{array}{l}\approx \\
8 \\
8\end{array}$ & $\begin{array}{l}\vec{m} \\
\tilde{g}\end{array}$ & 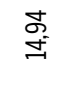 & $\begin{array}{l} \pm \\
8 \\
8\end{array}$ & $\left|\begin{array}{l}\mathscr{2} \\
\infty \\
\tilde{D}\end{array}\right|$ & $\begin{array}{l}\stackrel{\mathscr{m}}{\vec{F}} \\
\vec{\nabla}\end{array}$ & $\begin{array}{l}\text { t } \\
\text { 寸 }\end{array}$ & 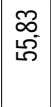 & 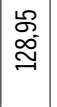 & $\begin{array}{l}\vec{G} \\
\vec{\nabla}\end{array}$ & $\begin{array}{l}\mathscr{O} \\
\stackrel{2}{2}\end{array}$ & $\begin{array}{l}2 \\
8 \\
8\end{array}$ & 8 \\
\hline$\stackrel{\Omega}{=}$ & 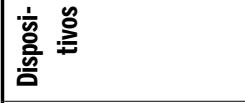 & $\begin{array}{l}\mathscr{m} \\
\infty \\
0\end{array}$ & $\begin{array}{l}8 \\
0 \\
0 \\
\sim\end{array}$ & $\begin{array}{l}\widetilde{O} \\
\stackrel{\sim}{\sim}\end{array}$ & $\begin{array}{l}\stackrel{8}{0} \\
\text { ்ָ }\end{array}$ & 吾 & $\underset{\tilde{d}}{\stackrel{\overbrace{}}{\sim}}$ & 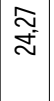 & $\underset{\sim}{\infty}$ & 孨 & 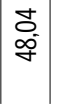 & 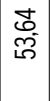 & $\underset{-i}{\stackrel{s}{*}}$ & 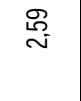 & 8 \\
\hline 읃 & 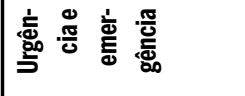 & $\begin{array}{l}\vec{\sigma} \\
\infty \\
\infty\end{array}$ & $\begin{array}{l}\mathscr{N} \\
\stackrel{\sim}{\sim}\end{array}$ & 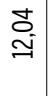 & ஜֶ. & $\begin{array}{l}\text { 足 } \\
\text { d }\end{array}$ & $\mid \begin{array}{l}0 \\
\text { 엄 } \\
\text { | }\end{array}$ & 勇 & 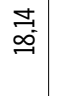 & 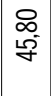 & $\stackrel{m}{\stackrel{m}{=}}$ & \begin{tabular}{l} 
\pm \\
\multirow{Z}{*}{}
\end{tabular} & \begin{tabular}{c}
$\infty$ \\
\multirow{+}{*}{}
\end{tabular} & $\begin{array}{l}\vec{\infty} \\
\underset{\Im}{\sim}\end{array}$ & 8 \\
\hline 즌 & 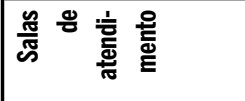 & \begin{tabular}{l}
$\stackrel{2}{0}$ \\
\hdashline \\
\hdashline
\end{tabular} & 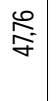 & $\stackrel{\dddot{m}}{\stackrel{0}{0}}$ & ঙ্ల్ల & $\begin{array}{l}\mathscr{m} \\
\vec{F}\end{array}$ & $\begin{array}{l}\tilde{m} \\
\stackrel{f}{*}\end{array}$ & $\begin{array}{c}0 \\
6 \\
6 \\
5\end{array}$ & 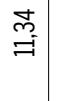 & $\begin{array}{l}\vec{\sigma} \\
\vec{m}\end{array}$ & $\left|\begin{array}{c}0 \\
0 \\
0 \\
m\end{array}\right|$ & $\frac{d}{0}$ & $\underset{f}{q}$ & $\underset{\sim}{\ddot{*}}$ & 8 \\
\hline Ф & 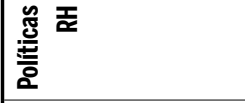 & $\stackrel{2}{\stackrel{2}{*}}$ & $\begin{array}{l}\widetilde{\Omega} \\
\stackrel{\infty}{\infty}\end{array}$ & న్స & $\frac{\widetilde{\sigma}}{\widetilde{\sigma}}$ & $\begin{array}{l}\text { 守 } \\
\text { 字 }\end{array}$ & $\mid \begin{array}{l}\tilde{z} \\
\tilde{0} \\
0\end{array}$ & 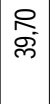 & $\underset{8}{\stackrel{8}{0}}$ & $\frac{\widetilde{\Xi}}{\tilde{m}}$ & 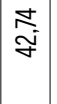 & 衤 & 吕 & 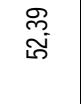 & 8 \\
\hline $\begin{array}{l}\text { ठ } \\
\text { i } \\
\text { s) }\end{array}$ & 这 $\frac{0}{0}$ & $\frac{9}{9}$ & $\begin{array}{l}\text { के } \\
\text { త్ }\end{array}$ & $\begin{array}{l}\stackrel{+}{\circ} \\
\stackrel{N}{2}\end{array}$ & $\stackrel{\infty}{\stackrel{\sim}{\sim}}$ & $\begin{array}{l}8 \\
\qquad \\
\bar{m}\end{array}$ & 足 & 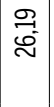 & $\begin{array}{l}8 \\
\stackrel{8}{ }\end{array}$ & 总 & 点 & 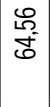 & $\underset{9}{\stackrel{9}{*}}$ & $\begin{array}{l}\vec{\sigma} \\
\ddot{\sim}\end{array}$ & 8 \\
\hline.$\Xi$ & 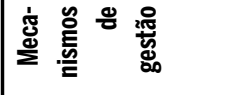 & \begin{tabular}{l}
$\stackrel{2}{\sim}$ \\
\multirow{F}{*}{}
\end{tabular} & $\begin{array}{l}\stackrel{0}{\infty} \\
\text { m }\end{array}$ & $\stackrel{\stackrel{2}{\sigma}}{\sigma}$ & 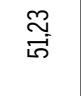 & $\begin{array}{l}\widetilde{O} \\
\stackrel{\sim}{\sim}\end{array}$ & $\mid \begin{array}{l}m \\
\tilde{s}\end{array}$ & $\begin{array}{l}z \\
0 \\
\sim\end{array}$ & $\stackrel{m}{\stackrel{m}{\sim}}$ & 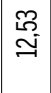 & $\begin{array}{l}8 \\
0 \\
0 \\
0\end{array}$ & 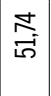 & $\begin{array}{l}8 \\
\stackrel{8}{*}\end{array}$ & $\stackrel{\infty}{\rightarrow}$ & 8 \\
\hline 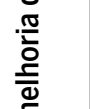 & 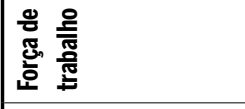 & 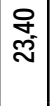 & $\begin{array}{l}\infty \\
0 \\
0 \\
0\end{array}$ & $\begin{array}{l}\mathbb{8} \\
\mathbb{0} \\
\stackrel{0}{0}\end{array}$ & 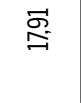 & 胥 & 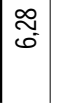 & $\begin{array}{l}\mathscr{n} \\
\sigma \\
\sigma\end{array}$ & $\underset{8}{\stackrel{8}{0}}$ & \begin{tabular}{|l|}
2 \\
0 \\
0 \\
0
\end{tabular} & $\begin{array}{l}\stackrel{2}{2} \\
\stackrel{2}{S}\end{array}$ & 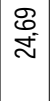 & $\underset{-i}{8}$ & $\stackrel{\overparen{\Xi}}{\leftrightarrows}$ & 8 \\
\hline 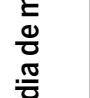 & 产 旁 & $\begin{array}{l}\stackrel{8}{2} \\
\text { ले }\end{array}$ & $\begin{array}{l}\widetilde{\sigma} \\
\text { }\end{array}$ & 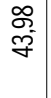 & 竎 & $\begin{array}{l}\text { F } \\
\text { क् } \\
\text { p }\end{array}$ & $\underset{\tilde{n}}{\mathfrak{F}}$ & 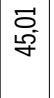 & 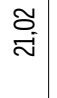 & 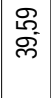 & $\begin{array}{l}\tilde{\tilde{E}} \\
\tilde{\tilde{n}}\end{array}$ & \begin{tabular}{|l|}
2 \\
0 \\
$q$ \\
$q$
\end{tabular} & $\underset{\vec{N}}{\mathbb{N}}$ & 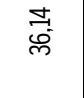 & 8 \\
\hline $\begin{array}{l}z \\
0 \\
\frac{\pi}{\pi} \\
\frac{\pi}{\pi} \\
\frac{\pi}{z}\end{array}$ & 迹 을 & 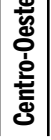 & 浮 & 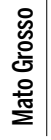 & 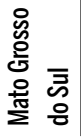 & 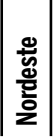 & 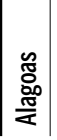 & 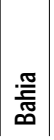 & 营 & 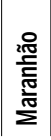 & 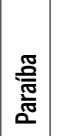 & 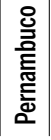 & 产 & 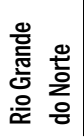 & 高 \\
\hline
\end{tabular}




\begin{tabular}{|c|c|c|c|c|c|c|c|c|c|c|c|c|c|c|c|c|}
\hline $\begin{array}{l}\stackrel{8}{G} \\
\text { 列 }\end{array}$ & 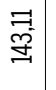 & 응 & $\begin{array}{l}\widetilde{\Xi} \\
\text { ऽ్ }\end{array}$ & $\mid$\begin{tabular}{c|}
$\mathscr{8}$ \\
$\stackrel{5}{\infty}$ \\
$\infty$
\end{tabular} & 8 & 8 & $\begin{array}{l}\text { m్t } \\
\infty \\
\text { : }\end{array}$ & $\underset{\infty}{\widetilde{\infty}}$ & $\begin{array}{l}\vec{\nexists} \\
\vec{m} \\
\ddot{m}\end{array}$ & 임 & $\begin{array}{l}\text { के } \\
\text { गे. } \\
\infty\end{array}$ & \begin{tabular}{l}
$\mathscr{0}$ \\
0 \\
\hdashline \\
\hdashline
\end{tabular} & 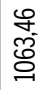 & 웅 & 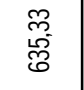 & 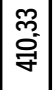 \\
\hline $\begin{array}{l}\stackrel{2}{G} \\
\text { a }\end{array}$ & $\underset{\leftarrow}{\widetilde{5}}$ & 응 & 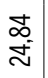 & $\stackrel{\stackrel{\infty}{\infty}}{\sim}$ & 응 & 응 & 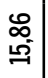 & $\stackrel{\dddot{m}}{=}$ & $\underset{\exists}{\exists}$ & 응 & 음 & $\begin{array}{l}\sqrt[5]{2} \\
\text { 亗 }\end{array}$ & $\begin{array}{l}\hat{m} \\
\tilde{m}^{-}\end{array}$ & 8 & 㣽 & ஜ্ণ \\
\hline 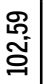 & $\begin{array}{l}\infty \\
\stackrel{\infty}{\vdots} \\
\stackrel{\Omega}{\sim}\end{array}$ & 응 & 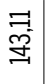 & $\begin{array}{l}\overrightarrow{0} \\
00 \\
0\end{array}$ & 8. & 응 & 을 & $\underset{\sim}{\mathbb{N}}$ & $\begin{array}{l}2 \\
8 \\
8\end{array}$ & 8 & $\underset{\sim}{\stackrel{\sim}{\sim}}$ & 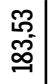 & 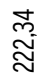 & 8 & $\begin{array}{l}\frac{0}{0} \\
\frac{1}{0}\end{array}$ & 忢 \\
\hline 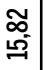 & $\underset{m}{\stackrel{N}{m}}$ & 응 & 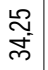 & 8 & 8 & 8 & $\underset{\widetilde{\Xi}}{\approx}$ & $\underset{ت}{\stackrel{\Xi}{二}}$ & $\underset{\widetilde{m}}{\widetilde{\pi}}$ & 응 & 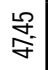 & 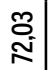 & $\frac{\tilde{\Omega}}{\infty}$ & 응 & 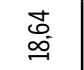 & $\begin{array}{l}\text { पे } \\
\text { क्. }\end{array}$ \\
\hline $\begin{array}{l}\text { g. } \\
\text { ㄱ. }\end{array}$ & $\begin{array}{l}\text { D } \\
\text { ల్ }\end{array}$ & 8 & $\begin{array}{l}\mathscr{O} \\
\mathscr{g} \\
\mathscr{q}\end{array}$ & $\begin{array}{c}\text { D } \\
=\end{array}$ & 8 & 응 & 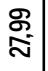 & $\ddot{\mathscr{O}}$ & $\begin{array}{l}\hat{g} \\
\text { ơ }\end{array}$ & 8 & $\begin{array}{l}\stackrel{\mathscr{a}}{\mathbf{m}} \\
\vec{m}\end{array}$ & 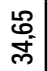 & 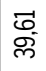 & 응 & $\begin{array}{l}\sqrt{5} \\
\stackrel{9}{9}\end{array}$ & \begin{tabular}{l}
$\stackrel{2}{m}$ \\
\multirow{\sim}{\sim}{}
\end{tabular} \\
\hline$\underset{\beth}{\mathbb{Z}}$ & $\underset{-}{\infty}$ & 응 & $\begin{array}{l}\text { வூ } \\
\text { న్య }\end{array}$ & $\stackrel{m}{\stackrel{m}{\sim}}$ & 8 & ㅇ. & 농 & 8 & $\underset{\varpi}{\widetilde{\Xi}}$ & 8 & 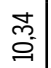 & $\underset{\mathbb{N}}{\vec{N}}$ & 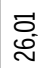 & 응 & $\begin{array}{l}\infty \\
\text { ల్ } \\
\underline{\emptyset}\end{array}$ & $\begin{array}{l}2 \\
2 \\
g\end{array}$ \\
\hline 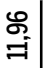 & $\begin{array}{c}\infty \\
\infty \\
\infty\end{array}$ & 8 & $\frac{\infty}{\check{D}}$ & $\stackrel{\stackrel{\sim}{\simeq}}{\cong}$ & 8 & 응 & $\stackrel{9}{\mathbb{S}}$ & $\begin{array}{l}\mathscr{g} \\
\mathrm{O}\end{array}$ & $\underset{ㅁ}{\vec{S}}$ & 응 & \begin{tabular}{l}
$\infty$ \\
$\stackrel{0}{ \pm}$ \\
\multirow{J}{*}{}
\end{tabular} & $\begin{array}{l}\mathbb{Z} \\
6 \\
6\end{array}$ & $\begin{array}{l}\hat{\sigma} \\
\tilde{\sigma}\end{array}$ & 8 & $\begin{array}{l}\underset{\infty}{\approx} \\
\underset{\sigma}{\approx}\end{array}$ & 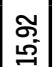 \\
\hline $\begin{array}{l}\text { กิ } \\
\text { sิ }\end{array}$ & $\begin{array}{l}\hat{\theta} \\
\mathscr{8}\end{array}$ & 웅 & 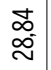 & 寽 & 응 & 응 & $\underset{\widetilde{f}}{\mathcal{f}}$ & 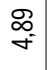 & $\begin{array}{c}\text { 点 } \\
\text { 员 }\end{array}$ & 8 & $\begin{array}{l}\infty \\
\vdots \\
b\end{array}$ & 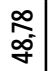 & હి & 응 & 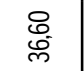 & $\begin{array}{l}\widetilde{S} \\
\dot{\sim}\end{array}$ \\
\hline$\widetilde{\Xi}$ & 8 & 8 & $\underset{ন}{\stackrel{\Xi}{二}}$ & $\mid \begin{array}{l}\tilde{\Xi} \\
\infty \\
\infty\end{array}$ & 8 & 응 & 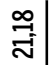 & 8 & 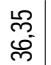 & 8 & $\begin{array}{c}\overrightarrow{2} \\
\stackrel{2}{2}\end{array}$ & 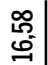 & 莺 & 8 & 崫 & $\begin{array}{l}\infty \\
0 \\
-1\end{array}$ \\
\hline$\stackrel{\overbrace{}}{\approx}$ & $\begin{array}{l}\text { S } \\
\text { g. }\end{array}$ & 8 & $\begin{array}{l}\vec{E} \\
\mathbb{e}\end{array}$ & $\frac{2}{\sigma}$ & 8 & 응 & $\begin{array}{l}\stackrel{0}{\infty} \\
= \\
=\end{array}$ & 足 & 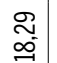 & 8 & $\stackrel{m}{\Xi}$ & న్ & $\stackrel{\infty}{\stackrel{\infty}{\sim}}$ & 응 & $\stackrel{\sqrt{\rho}}{=}$ & m \\
\hline $\begin{array}{l}\text { வ } \\
\text { ָే }\end{array}$ & $\begin{array}{l}0 \\
\stackrel{0}{n} \\
\infty \\
\infty\end{array}$ & 응 & 寻 & 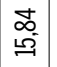 & 8 & 응 & $\begin{array}{l}\text { 용 } \\
\text { o }\end{array}$ & s̆. & $\underset{:}{\stackrel{2}{6}}$ & 8 & 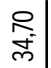 & స్ & $\frac{e}{\tilde{\sim}}$ & 용 & 䍘 & $\left|\begin{array}{l}0 \\
0 \\
0 \\
0\end{array}\right|$ \\
\hline 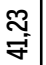 & $\begin{array}{l}\mathfrak{F} \\
\tilde{g}\end{array}$ & 응 & $\underset{\mathbb{N}}{\mathbb{N}}$ & 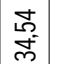 & 8 & 응 & $\begin{array}{l}0 \\
0 \\
0 \\
0\end{array}$ & 8 & 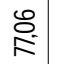 & 응 & $\begin{array}{l}\infty \\
\delta 8 \\
\delta 8\end{array}$ & 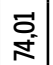 & $\begin{array}{l}\stackrel{p}{0} \\
\infty \\
\infty\end{array}$ & ㅇ. & $\begin{array}{l}\text { \& } \\
\text { \&े }\end{array}$ & 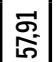 \\
\hline $\begin{array}{l}\mathbf{E} \\
\dot{\tilde{e}}\end{array}$ & \begin{tabular}{|c|}
$\infty$ \\
0 \\
$\tilde{N}$
\end{tabular} & 응 & $\begin{array}{l}\underset{F}{*} \\
\vec{F}\end{array}$ & $\begin{array}{l}0 \\
\stackrel{p}{\tilde{m}}\end{array}$ & 8 & 웅 & 号 & $\underset{m}{\stackrel{9}{m}}$ & 夆 & 8 & $\begin{array}{c}\vec{Z} \\
\underset{\infty}{\infty}\end{array}$ & $\begin{array}{l}\stackrel{2}{\sim} \\
\stackrel{\sim}{m}\end{array}$ & 萑 & 응 & 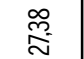 & $\begin{array}{l}\simeq \\
\tilde{m}\end{array}$ \\
\hline 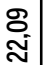 & 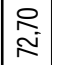 & 8 & $\begin{array}{l}\stackrel{D}{0} \\
\tilde{m}\end{array}$ & $\stackrel{\stackrel{2}{\leftrightarrows}}{\leftrightarrows}$ & 8 & 응 & $\underset{\tilde{m}}{\tilde{m}}$ & $\begin{array}{l}\vec{\sigma} \\
\dot{\sigma}\end{array}$ & $\begin{array}{c}\hat{7} \\
\text { o } \\
\text { o }\end{array}$ & 8 & $\begin{array}{c}\stackrel{8}{7} \\
6 \\
6\end{array}$ & $\begin{array}{l}\tilde{\sigma} \\
\tilde{0} \\
0\end{array}$ & $\underset{\tilde{\sigma}}{\approx}$ & ㅇ. & $\frac{9}{9}$ & 岕 \\
\hline న్సి & 호 & 응 & $\underset{\Xi}{\stackrel{\Xi}{\Im}}$ & 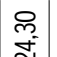 & 8 & 응 & $\overline{0}$ & $\underset{-}{\mathbb{J}}$ & 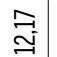 & 8 & $\begin{array}{c}\mathscr{D} \\
\infty \\
\infty\end{array}$ & $\begin{array}{l}0 \\
0 \\
\dot{m}\end{array}$ & $\begin{array}{l}{ }^{2}{ }^{\circ} \\
6 \\
0\end{array}$ & 응 & $\frac{\infty}{\sigma^{2}}$ & $\mid$ \\
\hline $\begin{array}{l}\mathscr{d} \\
\text { న }\end{array}$ & $\begin{array}{l}\underset{F}{F} \\
\underset{\forall}{*}\end{array}$ & 8 & $\begin{array}{l}\stackrel{\mathscr{R}}{\tilde{g}} \\
\stackrel{7}{*}\end{array}$ & ơ & 8 & 8 & ָ̃. & 8 & $\begin{array}{l}\infty \\
\stackrel{\infty}{0} \\
\stackrel{p}{0}\end{array}$ & 8 & $\begin{array}{l}\mathscr{D} \\
\$ \\
\dot{y}\end{array}$ & 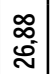 & 官 & 웅 & $\begin{array}{l}\widetilde{0} \\
\text { 品 }\end{array}$ & 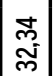 \\
\hline$\frac{d}{\frac{0}{0}}$ & 离 & 㺃 & 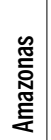 & 管 & 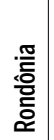 & 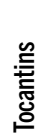 & 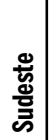 & 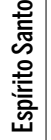 & 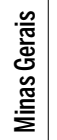 & 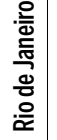 & 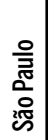 & $\bar{\Xi}$ & 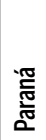 & 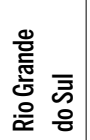 & 焉 & 홍 \\
\hline
\end{tabular}




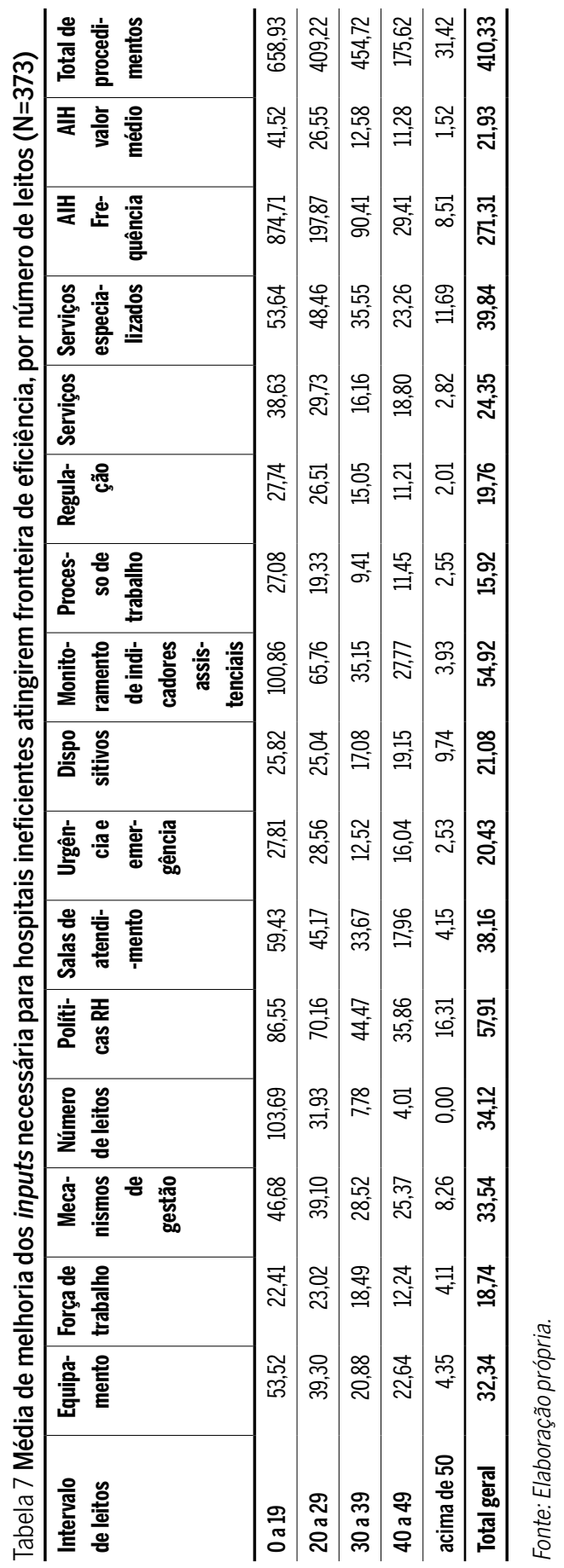


as variáveis. Na região Centro-Oeste o percentual de melhoria para serviços especializados e de AIH frequência é maior entre os hospitais públicos estaduais; na região Nordeste os hospitais privados com fins lucrativos demandam maior investimento para melhoria da regulação, serviços e total de procedimentos; na região norte para quase totalidade das variáveis os hospitais públicos estaduais precisam de ajuste maior do que os demais (exceto para as variáveis de dispositivo, regulação e total de procedimentos); na região Sudeste é maior a necessidade de incremento dos hospitais privados sem fins lucrativos para o total de procedimentos, e na região Sul também os privados com fins lucrativos demonstram carência maior de melhoria para força de trabalho, regulação, serviços e total de procedimentos.

\section{Discussão}

A aplicação do modelo de eficiência traduz um paradoxo típico de estudos dessa natureza, pois representam $25,27 \%$ da amostra inicial de referência (2.777 hospitais participaram do estudo e pela natureza técnica permaneceram somente os que não apresentaram variáveis com valor igual a zero) e ao mesmo tempo uma base com um quantitativo inédito de unidades entre os estudos nacionais. Essa condição consolida a confiabilidade dos dados pela amplitude da frequência de todas as variáveis estudadas no modelo de eficiência. O Quadro 4 apresenta a síntese do perfil e da caracterização geral do total de hospitais de pequeno porte que tiveram sua eficiência avaliada pela análise envoltória de dados.

A distribuição pelas regiões e estados brasileiros, com predominância no Nordeste, evidencia a prerrogativa histórica no país da construção de hospitais de pequeno porte para suprir carências regionais de saúde, inclusive como mecanismo político em municípios, por exemplo, com baixa densidade populacional. Segundo Ugá e López (2007, p. 926, “[a] criação de pequenos hospitais, principalmente em municípios do interior, deve-se aos mecanismos de investimento tradicionais no Brasil, particularmente às emendas parlamentares que, raras vezes, têm em consideração as necessidades de saúde mais prementes da localidade".

Esse ponto é importante, uma vez que a amostra utilizada como base do modelo de eficiência replica a distribuição geral dos HPPs no Brasil, com exceção das regiões Norte e Sudeste, que possuem percentual menor de 
hospitais na distribuição geral, mas foram as regiões com maior percentual de hospitais eficientes. Chama a atenção o fato de que a região Sudeste foi aquela com maior redução dos hospitais da amostra inicial para a amostra do estudo, ou seja, onde ocorreu maior frequência de hospitais com respostas insuficientes nas variáveis que foram escolhidas para o modelo de eficiência.

Quadro 4 Síntese da caracterização geral de todos os hospitais

\begin{tabular}{|c|c|c|c|}
\hline Item & $\begin{array}{r}\text { Amostra do estudo em tela } \\
\text { (antes do modelo } \\
\text { de eficiência) }\end{array}$ & $\begin{array}{l}\text { Hospitais } \\
\text { Eficientes }\end{array}$ & $\begin{array}{r}\text { Hospitais } \\
\text { Ineficientes }\end{array}$ \\
\hline Total de unidades & 702 hospitais & 329 unidades $(46,87 \%)$ & 373 unidades $(53,13 \%)$ \\
\hline Distribuição & $\begin{array}{r}\text { Maior frequência de } \\
\text { hospitais na região Nordeste }\end{array}$ & $\begin{array}{r}\text { Norte e Sudeste } \\
(62,50 \% \text { e } 56,16 \% \\
\text { respectivamente) }\end{array}$ & $\begin{array}{r}\text { Centro-0este e Sul } \\
(58,46 \% \text { e } 63,57 \%, \\
\text { respectivamente) }\end{array}$ \\
\hline Número de leitos & $\begin{array}{r}\text { Maior frequência de } \\
\text { hospitais com } 20 \text { a } 29 \text { leitos }\end{array}$ & $\begin{array}{r}\text { Maior frequência de } \\
\text { hospitais com } 30 \text { a } 39 \text { leitos }\end{array}$ & $\begin{array}{r}\text { Maior frequência de } \\
\text { hospitais com } 20 \text { a } 29 \text { leitos }\end{array}$ \\
\hline Esfera de gestão & $\begin{array}{l}\text { Maior frequência de hospitais } \\
\text { de gestão pública municipal }\end{array}$ & $\begin{array}{r}\text { Maior frequência de hospitais } \\
\text { privados com e sem fins } \\
\text { lucrativos e públicos estaduais }\end{array}$ & $\begin{array}{l}\text { Maior frequência de hospitais } \\
\text { de gestão pública municipal }\end{array}$ \\
\hline
\end{tabular}

Fonte: Elaboração própria.

A variedade quanto à distribuição dos hospitais de pequeno porte, com o predomínio daqueles da esfera pública, reforça a ausência de mecanismos convergentes com diretrizes mais amplas do sistema de saúde. Esse desenho corrobora, em certa medida, a organização do sistema público de saúde, que define em sua operacionalização a descentralização da gestão da saúde para o ente municipal. Existe na literatura um relativo consenso sobre os avanços dessa descentralização no que diz respeito não apenas às transferências de serviços, mas também de responsabilidades, poder e recursos da esfera federal para as esferas estadual e municipal (Levcovitz; Lima; Machado, 2001). Para Silva (2004), o processo de descentralização na saúde é acompanhado de problemas relacionados à redefinição de competências entre as esferas de governo e apresenta um grande desafio no que diz respeito à coordenação das relações intergovernamentais, pois se constata a presença de relações competitivas entre as instâncias de governo, embora seja maior a influência por um modelo cooperativo para práticas de políticas públicas. 
Nesse mesmo sentido, o fato de a gestão municipal representar o maior contingente de instituições faz relembrar a reflexão já amplamente debatida quanto ao aperfeiçoamento das redes de atenção à saúde como mecanismo de gestão importante no contexto brasileiro com vistas à capacidade resolutiva do sistema.

O modelo aplicado para os HPPs possibilita analisar inputs e outputs simultaneamente para impacto na eficiência. O score de cada categoria, em termos percentuais, demonstra o acréscimo mínimo necessário para o conjunto das variáveis para o alcance da fronteira de eficiência. A análise é feita em dois agrupamentos principais: o primeiro com base no score geral de melhoria que cada categoria apresentou, e o segundo, a partir de uma síntese por região com base no número de leitos, natureza jurídica e na classificação como eficiente ou não eficiente. Essa sistematização busca organizar a sequência do texto, mas é importante reforçar que as diferentes variáveis estão inter-relacionadas e que o conteúdo é cumulativo em toda a sua extensão para compreensão mais abrangente e integrada. $\bigcirc$ Quadro 5 apresenta o percentual necessário de melhoria, para os hospitais de pequeno porte, para cada dimensão do modelo de eficiência à luz da classificação proposta a partir de Donabedian (1992).

Quadro 5 Melhoria necessária para eficiência dos hospitais por dimensão e categorias

\begin{tabular}{l|r|l|r|l|r}
\hline Estrutura (inputs) & $\%$ & Processos (inputs) & $\%$ & Resultados (outputs) & \% \\
\hline Políticas GRH & 57,91 & $\begin{array}{l}\text { Monitoramento de } \\
\text { indicadores assistenciais }\end{array}$ & 54,92 & Total de procedimentos & 410,33 \\
\hline Salas de atendimento & 38,16 & Serviços especializados & 39,84 & Frequência AlH & 271,31 \\
\hline Leitos disponíveis & 34,12 & Serviços & 24,35 & Valor médio de AlH & 21,93 \\
\hline Mecanismos de gestão & 33,54 & Dispositivos & 21,08 & & \\
\hline Equipamentos & 32,34 & Regulação & 19,76 & & \\
\hline Urgência e emergência & 20,43 & $\begin{array}{l}\text { Processo de trabalho/ } \\
\text { procedimentos }\end{array}$ & 15,92 & & \\
\hline Força de trabalho & 18,74 & & & & \\
\hline
\end{tabular}

Fonte: Elaboração própria.

A melhoria da eficiência dos hospitais de pequeno porte é impactada fortemente pela dimensão de resultados, num segundo patamar pela dimensão de estrutura, e por último, pela dimensão de processos. $O$ detalhamento de cada categoria é feito a seguir em ordem decrescente quanto ao percentual de melhoria necessário e/ou pela interdependência dos conteúdos. 
Pela perspectiva dos resultados (outputs) é significativa a necessidade do aumento da quantidade de procedimentos realizados pelos HPPs e de AIHs emitidas (visto que uma $\mathrm{AIH}$ pode registrar vários procedimentos). No entanto, o valor médio pago por AIH tem uma contribuição mais periférica para a melhoria da eficiência, o que distancia a dependência "exclusiva" de recursos financeiros tão frequentemente anunciada como condição para qualidade dos resultados de instituições hospitalares. Embora seja questão relevante para o contexto da gestão hospitalar, seu efeito está equiparado às dimensões com menor impacto para a eficiência.

O fator produtividade, com maior necessidade de ajuste para alcance da eficiência, pode ter peso preponderante quanto ao papel desses equipamentos de saúde, pois remete a discussão recorrente quanto às maneiras eficientes de utilizar e alocar os recursos em função das limitações de financiamento, por exemplo, (Aktaş et al., 2007). Adicionalmente ao desafio da gestão dos recursos financeiros, existem pressões para a redução de custos aliada ao incremento de qualidade dos serviços prestados nos hospitais provenientes de órgãos governamentais, seguradoras de planos de saúde, da comunidade e dos pacientes (Li; Benton, 1996).

A partir do modelo de eficiência, embora não seja possível isolar a contribuição de uma variável em particular, vale destacar a capacitação para profissionais médicos, de enfermagem e demais categorias, para o conteúdo aplicado ao modelo assistencial vigente. Lins et al. (2007, p. 996), em estudo $^{6}$ com aplicação da metodologia DEA em 31 hospitais universitários brasileiros, observa que a transformação de ações punitivas em ações educativas e formativas é condição básica para melhoria de oferta e saúde para a população. $O$ investimento financeiro necessário para a capacitação dos profissionais da saúde pode ser um desafio, especialmente entre os hospitais públicos, além da grande pulverização das ações que nem sempre ocorrem de forma coordenada e com foco nas necessidades efetivas do próprio serviço de saúde. A educação permanente, com foco na formação inserida no processo de trabalho, tem sido um modelo amplamente adotado pelas instituições de saúde, embora com participação majoritária dos profissionais de enfermagem.

De forma geral, o modelo de eficiência, pela quantidade de hospitais e variáveis consideradas, evidenciou resultados expressivos frente às la-

6 A literatura que utiliza DEA é escassa para variáveis na dimensão de ensino nos hospitais. 
cunas teóricas que acompanham essas temáticas, além do forte potencial para subsidiar políticas públicas ou o aperfeiçoamento da gestão das instituições que fazem parte da amostra do estudo. A análise dos resultados sugere reflexões sobre a lógica do sistema de saúde e a inserção dos HPPs, ao mesmo tempo que indica possíveis impactos de intervenção ou atuação em pontos específicos da gestão desses hospitais.

A análise sobre eficiência e ineficiência revelou aspectos que podem ajudar na compreensão da dinâmica hospitalar em municípios brasileiros, notadamente quando observados em suas peculiaridades locais e até mesmo estaduais. Sua contribuição para lançar luz à hipótese que norteou o trabalho de que a Gestão de Recursos Humanos contribui para o resultado dos hospitais de pequeno porte. No entanto, vale relembrar a ponderação de que a complexidade que circunda a eficiência hospitalar é tão grande que é impraticável estabelecer exatamente todos os indicadores e mensurar todas as dimensões possíveis que influenciam direta ou indiretamente na avaliação de desempenho de uma organização (Cunha, 2011).

Ainda sobre a avaliação em organizações de saúde tem-se como base o pressuposto de que as análises não podem ser exclusivamente quantitativas, isto é, elas dependem também de elementos qualitativos para sua avaliaçã, Some-se a isso a possibilidade de que as medidas de desempenho possam marginalizar interesses alheios, construindo realidades falsas e deixando de lado aspectos de relevância para a avaliação da organização de saúde (Cunha, 2011).

\section{Referências}

AKTAŞ, E.; ÜLENGIN, F.; ŞAHIN, S. Ö. A Decision support system to improve the efficiency of resource allocation in healthcare management. Socio Economic Planning Sciences, v. 41, n. 2, pp. 130-146, 2007.

ANDRADE, M. V; MAIA, A. C. Análise de eficiência hospitalar no estado de Minas Gerais. Belo Horizonte: CEDEPLAR/UFMG, 2007. Relatório Técnico.

COOK, W. D.; SEIFORD, L. M. Data Envelopment Analysis (DEA). Thirty years. European Journal of Operational Research, v. 192, n. 1, pp. 1-17, 2009.

CUNHA, J. A. C. Avaliação de desempenho e eficiência em organizações de saúde: um estudo em hospitais filantrópicos. 2011. Tese (Doutorado) - FEA/USP, São Paulo, 2011.

DONABEDIAN, A. The definition of quality and approaches to its assessment. Ann Arbor, Michigan: The University of Michigan Health Administration Press, 1980. V. I. 
DONABEDIAN, A. The role of outcomes in quality assessment and assurance. Quality Rev Bulletin, Nov, 18(11):356-60, 1992.

FACCHINI, L. A., BARBOSA, A. C. Q. et al. Estudo de hospitais de pequeno porte brasileiros. Diagnóstico, avaliação e espacialização. Belo Horizonte/Pelotas: UFPel; Observatório de Recursos Humanos em Saúde da FACE/UFMG, 2014. (Relatório de Pesquisa).

GLOUBERMAN S, et al. Managing the care of health and curing the Disease, Part I: Differentiation. Healthcare Management Review, p. 56-69, 2001.

GROSSKOPF, S.; VALDMANIS, V. Measuring hospital performance: a non-parametric approach. Journal of Health Economics, v. 6, n. 2, pp. 89-107, 1987.

HUSELID, M. A. The impact of Human Resource Management practices on turnover, productivity, and corporate financial performance. Academy of Management Journal, v. 38, n. 3, p. 635-672, 1995.

HUSSEY, P.; DE VRIES, H.; ROMLEY, J.; WANG, M.; CHEN, S.; SHEKELLE, P.; MCGLYNN, E. A Systematic Review of Health Care Efficiency Measures. First published: 15 May 2009.

JACOBS, R. Alternative Methods to Examine Hospital Efficiency: Date Envelopment Analysis and Stochastic Frontier Analysis. Health Care Management Science, v. 4, n. 2, pp. 103115, 2001.

LEVCOVITZ, E.; LIMA, L. D.; MACHADO, C. V. Política de saúde nos anos 90: relações intergovernamentais e o papel das Normas Operacionais Básicas. Ciência e Saúde Coletiva, Rio de Janeiro, v. 6, n. 2, 2001.

LI L.X. and BENTO, W.C. Performance measurement criteria in health care organizations: review and future research directions. EJOR 93(3): 449-468, 1996.

LINS, M. E. et al. O uso da Análise Envoltória de Dados (DEA) para avaliação de hospitais universitários brasileiros. Ciência e Saúde Coletiva, v. 12(4):985-998, 2007.

LÓPEZ, E. M. Uma revisão do papel dos hospitais de pequeno porte no SUS. 2004. Dissertação (Mestrado) - ENSP/Fiocruz, Rio de Janeiro, RJ, 2004.

MAGNUSSEN, J. Efficiency measurement and the operationalization of hospital production. Health Services Research, v. 31(1), p. 21-37, 1996.

MACHADO, S. P.; KUCHENBECKER, R. Desafios e perspectivas futuras dos hospitais universitários no Brasil. Ciência e Saúde Coletiva, Rio de Janeiro, v. 12, n. 4, jul./ago., 2007.

MELLO, J. C. C.; MEZA, L.; GOMES, E. G.; NETO, L. Integrated System for Decision Support (SIAD) [Sistema Integrado de Apoio a Decisão]: A Software Package for Data Envelopment Analysis Model. Pesquisa Operacional, 25(3), p. 493-503, 2005.

NETO, G. V.; MALIK, A. M. Tendências na assistência hospitalar (debate). Ciência e Saúde Coletiva, Rio de Janeiro, v. 12 n. 4, jul./ago. 2007.

PAAUWE, J. HRM and performance: achievements, methodological issues and prospects. Journal of Management Studies, v. 46, n. 1, p. 129-142, 2009.

PURBEY, S.; MUKHERJEE, K.; BHAR, C. Performance measurement system for healthcare processes. International Journal of Productivity and Performance Management, v. 56 Iss: 3, pp. 241-251, 2007.

RODRIGUES, J. M. Gestão de recursos humanos e desempenho hospitalar na Perspectiva da 
eficiência: um estudo em hospitais brasileiros de pequeno porte, Belo Horizonte, FACE/ UFMG, 2016 (tese de doutorado).

RODRIGUES, J. M.; TORRES, H. O. G. Eficiência/desempenho hospitalar e resultados da gestão de recursos humanos - uma aproximação possível? Revista eletrônica Gestão \& Sociedade, v. 9, n. 24, p. 1.128-1.142, set./dez., 2015.

SEXTON, T. R.; SILKMAN, R.H.; HOGAN, A. J. Data envelopment analysis: Critique and extensions. In: SILKMAN, R.H. (Ed.). Measuring efficiency: an assessment of data envelopment analysis. Jossey-Bass, San Francisco, 1986. p. 73-105.

SILVA, A. C. Z. SUS: avanços e obstáculos no processo de descentralização e coordenação intergovernamental. Revista do Serviço Público, ano 55, n. 4, 2004.

UGÁ, M. A. D.; LÓPEZ, E. M. Os hospitais de pequeno porte e sua inserção no SUS. Ciênc. Saúde Coletiva, Rio de Janeiro, v. 12, n. 4, ago. 2007.

VEILLARD, J. et al. A performance assessment framework for hospitals: the WHO regional office for Europe PATH project. Int. J. Qual. Health Care, Dec., 17(6):487-496, 2005.

WENG, S. J. et al. An extended DEA model for hospital performance evaluation and improvement. Health Serv Outcomes Res Method, 9:39-53. DOI 10.1007/s10742-008-0042-y, 2009.

ZIGAN, K., FRASER, M., DESOMBRE, T. Intangible resources as performance drivers in European hospitals. International Journal of Productivity and Performance Management, v. 57, n. 1, p. 57-71, 2008.

\section{Sobre os autores}

Júnia Marçal Rodrigues-juniamarcal1975@gmail.com UNIMED-BH, Belo Horizonte, MG, Brasil. ORCID: https://orcid.org/0000-0001-6449-612X.

Allan Claudius Queiroz Barbosa - allan@ufmg.br Universidade Federal de Minas Gerais, Belo Horizonte, MG, Brasil. ORCID: https://orcid.org/0000-0003-1266-5168.

\section{Sobre 0 artigo}

Recebido em 25 de março de 2020. Aprovado em 14 de setembro de 2020. 